\title{
Ventralis intermedius nucleus anatomical variability assessment by MRI structural connectivity
}

\author{
Francisca Ferreira ${ }^{\mathrm{a}, \mathrm{b}, \mathrm{c}, *}$, Harith Akram ${ }^{\mathrm{b}}$, John Ashburner ${ }^{\mathrm{c}}$, Ludvic Zrinzo ${ }^{\mathrm{b}}$, Hui Zhang a,d, \\ Christian Lambert ${ }^{\mathrm{c}}$ \\ ${ }^{a}$ EPSRC Centre for Doctoral Training in Intelligent, Integrated Imaging in Healthcare (i4health), University College London, Gower Street, London WC1E 6BT, United \\ Kingdom \\ ${ }^{\mathrm{b}}$ Functional Neurosurgery Unit, Department of Clinical and Motor Neurosciences, UCL Institute of Neurology, Queen Square, WC1N 3BG London, United Kingdom \\ ${ }^{\mathrm{c}}$ Wellcome Centre for Human Neuroimaging, 12 Queen Square, London WC1N 3AR, United Kingdom \\ ${ }^{\mathrm{d}}$ Department of Computer Science and Centre for Medical Image Computing, University College London, Gower Street, London WC1E 6BT, United Kingdom
}

\section{A R T I C L E I N F O}

\section{Keywords:}

Connectivity

Functional neurosurgery

Tremor

Individualized targeting

Probabilistic tractography

\begin{abstract}
A B S T R A C T
The ventralis intermedius nucleus (Vim) is centrally placed in the dentato-thalamo-cortical pathway (DTCp) and is a key surgical target in the treatment of severe medically refractory tremor. It is not visible on conventional MRI sequences; consequently, stereotactic targeting currently relies on atlas-based coordinates. This fails to capture individual anatomical variability, which may lead to poor long-term clinical efficacy. Probabilistic tractography, combined with known anatomical connectivity, enables localisation of thalamic nuclei at an individual subject level. There are, however, a number of confounds associated with this technique that may influence results.

Here we focused on an established method, using probabilistic tractography to reconstruct the DTCp, to identify the connectivity-defined Vim (cd-Vim) in vivo. Using 100 healthy individuals from the Human Connectome Project, our aim was to quantify cd-Vim variability across this population, measure the discrepancy with atlasdefined Vim (ad-Vim), and assess the influence of potential methodological confounds.

We found no significant effect of any of the confounds. The mean cd-Vim coordinate was located within $1.88 \mathrm{~mm}$ (left) and $2.12 \mathrm{~mm}$ (right) of the average midpoint and $3.98 \mathrm{~mm}$ (left) and $5.41 \mathrm{~mm}$ (right) from the ad-Vim coordinates. cd-Vim location was more variable on the right, which reflects hemispheric asymmetries in the probabilistic DTC reconstructed. The method was reproducible, with no significant cd-Vim location differences in a separate test-retest cohort. The superior cerebellar peduncle was identified as a potential source of artificial variance.

This work demonstrates significant individual anatomical variability of the cd-Vim that atlas-based coordinate targeting fails to capture. This variability was not related to any methodological confound tested. Lateralisation of cerebellar functions, such as speech, may contribute to the observed asymmetry. Tractography-based methods seem sensitive to individual anatomical variability that is missed by conventional neurosurgical targeting; these findings may form the basis for translational tools to improve efficacy and reduce side-effects of thalamic surgery for tremor.
\end{abstract}

\begin{tabular}{llll}
\multicolumn{2}{l}{ Abbreviations } & DWI & Diffusion weighted imaging \\
AC & Anterior commissure & DTCp & Dentato-thalamo-cortical tract \\
AP & Anisotropic power & ED & Euclidean distance \\
CSF & Cerebrospinal fluid & EPI & Echo planar imaging \\
CTT & Cerebellothalamic tract & ET & Essential tremor \\
CT & Computed tomography & FA & Fractional anisotropy \\
DBS & Deep brain stimulation & FOD & Fibre orientation distribution \\
DTI & Diffusion tensor imaging & fMRI & Functional magnetic resonance imaging
\end{tabular}

\footnotetext{
* Corresponding author at: Functional Neurosurgery Unit, Department of Clinical and Motor Neurosciences, UCL Institute of Neurology, Queen Square, WC1N 3BG London, United Kingdom.

E-mail address: francisca.ferreira.16@ucl.ac.uk (F. Ferreira).
} 
FNIRT FMRIB s linear image registration tool

FSL FMRIB's Software Library

FWE Family-wise error

FWHM Full width at half-maximum

GFA Generalized fractional anisotropy

GM Grey matter

GPi Globus pallidus pars interna

GPU Graphics processing unit

HARDI High angular resolution diffusion imaging

hiFU High intensity focused ultrasound

HCP Human Connectome Project

iMRI Interventional magnetic resonance imaging

IPG Implantable pulse generator

L-DOPA levodopa

MCMC Markov chain Monte Carlo

MDWI Mean DWIs with B0 image

MER Microelectrode recording

MNI Montreal Neurological Institute

MRI Magnetic resonance imaging

MS Multiple sclerosis

PC Posterior commissure

PD Parkinson's disease

PICo Probabilistic index of connectivity

ROI Region of interest

SPM Statistic parametric mapping

SCP Superior cerebellar peduncle

T1w T1-weighted

TIV Total intracranial volume

VBM Voxel based morphometry

Vim Ventralis intermedius nucleus

Voa Ventralis oralis nucleus

WM White matter

\section{Introduction}

The ventralis intermedius nucleus (Vim) is a wedge-shaped thalamic nucleus (Fig. 1) that is thought to function as a hub for sensorymotor integration (Mai and Forutan, 2012). The Vim nomenclature is part of the Hassler classification and corresponds to the inferior aspect of the ventro-lateral (VLp) nucleus in the Hirai and Jones classification (Hassler et al., 1959; Hirai and Jones, 1989). It contains kinaesthetic neurons, somatotopically arranged along the medial-lateral axis (Asanuma et al., 1983; Strick, 1976; Vitek et al., 1996, 1994), that respond to contralateral muscles and joints (Gallay et al., 2008; Jones, 2007, 1985; Llinás et al., 2005; Sakai, 2013). Sensory information is conveyed to the ipsilateral cerebellum directly via the spinocerebellar tracts (mossy fibres) and indirectly via the inferior olive (climbing fibres). Cerebellar efferents arise from the deep cerebellar nuclei (dentate, interposed and fastigial nuclei); the cerebellothalamic tract (CTT) passes through the superior cerebellar peduncle (SCP) and decussates at midbrain level, forming the brachium conjunctivum. CTT axons pass through and around the anterior contralateral red nucleus without synapsing, terminating in Vim (Gallay et al., 2008). Efferent tracts arise from Vim and project principally to the primary motor cortex (M1) (Jones, 2007), with minor projections to the SMA, pre-SMA and premotor cortex (Sakai, 2013). Collectively, the pathway connecting dentate to motor cortex via the Vim is known as the dentato-thalamo-cortical pathway (DTCp).

Vim neurons are predisposed to oscillatory firing behaviour that gives rise to tremor (Llinás et al., 2005; Llinas, 1988; Steriade and Llinás, 1988), making this structure a key node in the cerebello-thalamocortical "tremor network" (Al-Fatly et al., 2019; Muthuraman et al., 2012). In essential tremor (ET), tremor results from dysfunction at the inferior olive and cerebellar nuclei. In contrast, tremor in Parkinson's disease (PD) is thought to emerge through basal ganglia dysfunction, where increased activity of inhibitory GABAergic neurons in the GPi projecting to the thalamus gives rise to the rhythmic bursting activity of thalamic neurons at tremor frequency (Llinás et al., 2005; Llinas, 1988). Whilst these projections, according to non-human primate studies, are primarily to the ventralis oralis anterior (Voa) and not Vim (Kuo and Carpenter, 1973), it is thought that the reticular nucleus potentiates these aberrant signals and relays them to other thalamic structures, specifically Vim, via interneurons (Huguenard and McCormick, 2007), where they are propagated to the motor and supplementary motor areas (DeLong and Wichmann, 2009), and may be modulated by the cerebellum (Duval et al., 2016). In both pathologies, the Vim is instrumental in synchronising and propagating abnormal tremor-generating oscillations (Duval et al., 2016; Raethjen and Deuschl, 2012). Consequently, in medically refractory tremor syndromes, the Vim has become a target for stereotactic treatment via deep brain stimulation (DBS) or lesioning techniques (Benabid et al., 1993, 1991; Benabid, 1989; Cury et al., 2017; Hariz et al., 2008; Hirai et al., 1983; Pollak et al., 1993).

However, the Vim is not visible on conventional MRI sequences and stereotactic targeting in functional neurosurgery typically estimates Vim position from standardised atlas coordinates (Schaltenbrand, 1977; Talairach, 1988). These are adapted to individual subjects by using visible anatomical landmarks, such as the mid-anterior and posterior commissural points. Whilst this method provides a reproducible way to identify Vim, it is unable to account for inter-individual variability (Akram et al., 2018). There is a wealth of evidence demonstrating that inter-individual, and often inter-hemispheric, anatomical variability is substantial, for example in histologically defined primary visual cortex (Amunts, 2000; Alho, 2011), amygdala (Amunts, 2005) or thalamic nuclei (Morel et al., 1997) with the latter work concluding "interindividual nuclear variations cannot easily be taken into account by standardization procedures"; success in stereotactic surgery is dependent on accurate
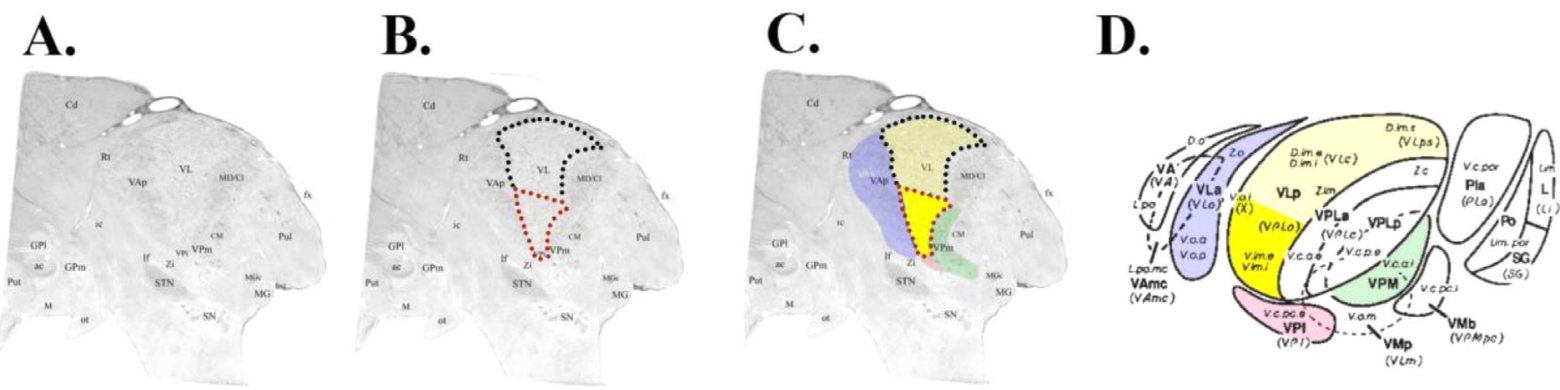

Fig. 1. A - Nissl-stained histological sagittal section of a human thalamus at $+10.75 \mathrm{~mm}$ from the midline; B - Outline of VL (dotted line) and position of Vim within VL on this atlas (red dotted line); C. Highlight of surrounding structures (bold yellow = Vim; pale yellow - VL; blue = Ventrolateral anterior; red - Ventroposterior inferior; green - Ventroposterior medial); D - Diagrammatic representation of lateral view of the thalamus showing relationships between thalamic nuclei, colourised as per C. A-C adapted with permission from Ilinsky et al. (2018). D adapted with permission from Gross et al. (2004). (For interpretation of the references to color in this figure legend, the reader is referred to the web version of this article.) 
targeting (Horn et al., 2019). To offset this limitation, surgical procedures are often performed with invasive mapping and the patient awake to allow target confirmation via neurophysiological techniques and /or micro- or macro-electrode stimulation. Such methods are uncomfortable for patients, rely on subjective measures and each surgical trajectory carries a small risk of intracranial haemorrhage leading to neurological deficit or death (Zrinzo et al., 2012). Moreover, intraoperative testing does not always guarantee good long-term tremor suppression as testing can be confounded by implantation oedema. Therefore, there is a clinical need to improve non-invasive pre-operative mapping of Vim to minimise unwanted side-effects, improve outcomes and increase comfort for patients (Chen et al., 2016).

The structural connectivity of Vim and the anatomical properties of DTCp have been proposed as a way to more accurately identify this structure in vivo using tractography-based methods (Akram et al., 2018; Coenen et al., 2011; Middlebrooks et al., 2018a; Pouratian et al., 2011; Sammartino et al., 2016; Tian et al., 2018). Tractography reconstructs white matter pathways of the brain using diffusion-weighted imaging (DWI), an MRI modality that measures the diffusive properties of water and has become a widely used tool to investigate the human brain (Mancini et al., 2019). DWI allows the estimation of local fibre orientations at each voxel location; individual white matter pathways can thus be traced by following these orientations from one voxel to the next. A number of methods using structural connectivity to identify Vim apply deterministic tractography but this technique has a number of major limitations (Coenen et al., 2011; Nimsky et al., 2016; Nowacki et al., 2018; Sammartino et al., 2016). It only provides a single tract estimate for each seed, based upon the principal diffusion direction, and is therefore unable to resolve complex fibre orientations such as fibre decussation (Abhinav et al., 2014), it underestimates the size and extent of tracts (Petersen et al., 2017), and generates unreliable results in grey matter regions (Behrens et al., 2003). Given the structural properties of the DTCp, it is clear these issues are highly relevant for Vim targeting. Probabilistic tractography represents an alternative approach as it is able to quantify the uncertainty of multiple fibre-orientations at a voxel-wise level and yields a higher level of precision compared to deterministic methods (Abhinav et al., 2014; Petersen et al., 2017). Several methods using probabilistic tractography to improve localisation and segmentation of thalamic nuclei have emerged (Akram et al., 2018; Behrens et al., 2003; Middlebrooks et al., 2018a; Pouratian et al., 2011; Traynor et al., 2010). While the translational implications for functional neurosurgery are clear, there are a number of confounds that could influence the results, such as head motion (Baum et al., 2018; Le Bihan et al., 2006; Ling et al., 2012; Tijssen et al., 2009) or pre-processing choices used to estimate white-matter connectivity and reconstruct tracts. A prerequisite for any translational tool designed to aid surgical decision making, is that it should provide an accurate representation of the true underlying anatomy; therefore, possible methodological confounds should be minimised or accounted for in the modelling.

Here we follow an established state-of-the-art method to identify Vim in vivo using probabilistic tractography. Studying 100 healthy individuals from the Human Connectome Project, we set out to quantify positional variability of Vim and compare tractography-determined coordinates to the surgical standard. We examine the impact of various methodological confounds, including participant motion and regional brain volumes, to establish whether the inter-subject variability reflects true variation in anatomy or simply methodological artefacts.

\section{Methods and materials}

\subsection{Ethics statement}

This study performed secondary analysis in anonymised human subjects. WU-Minn HCP data was obtained from consenting adults and their families and features that may identify an individual or family unit are safeguarded and not divulged.

\subsection{Data and code availability}

WU-Minn HCP data is freely available at https://humanconnectome. org. Derived data results and analysis code have been made available in an open repository https://github.com/qmaplab/vim_variability .

\subsection{Subjects}

One hundred healthy, unrelated individuals from the WU-Minn Human Connectome Project were studied (S100 dataset, Van Essen et al., 2012). We sub-selected the $S 100$ dataset for the main analysis as we felt it would be adequate to answer whether Vim positional variability, as defined using tractography, was significantly influenced by methodological confounds, and had the advantage of avoiding any potential issues due to familial structural covariance(Amico and Goñi, 2018). We also undertook a separate analysis using the test-retest HCP cohort to ensure the method was reproducible, and these results for this are reported in a separate section.

The HCP protocol is fully described in Glasser et al. (2013). In this work, we only use the T1w and DWI data from the minimally processed pipeline, and have therefore summarised the relevant subsections that apply to this work: the diffusion MRI underwent EPI distortion estimation using the 'topup' tool in FSL, with subsequent correction of EPI distortion, eddy current, motion and signal dropout in using the 'eddy' tool in FSL. The mean B0 image was also distortion corrected and a transform between native diffusion and structural space calculated (Glasser et al., 2013). In this work, we used the T1w MRI that had been aligned and resliced to $1.25 \mathrm{~mm}$ DWI space. The minimally processed pipeline provided all the required inputs (bvals, bvecs, nodif_brain_mask) for the additional processing steps outlined below.

\subsubsection{Diffusion MRI imaging protocol}

WU-Minn HCP adopt a multi-shell multi-band spin-echo EPI sequence with three different gradient tables, each table acquired once with right-to-left and left-to-right phase encoding polarities; each gradient table includes 90 diffusion weighting directions and six $\mathrm{b}=0$ acquisitions. Fourier 6/8, field of view (FOV) $210 \mathrm{~mm} \times 180 \mathrm{~mm}$, $\mathrm{TR}=5520 \mathrm{~ms}, \mathrm{TE}=89.5 \mathrm{~ms}$. 111 slices were acquired with $1.25 \mathrm{~mm}$ thickness and $1.5 \mathrm{~mm}$ in-plane resolution, and b-values of 1000,2000 and $3000 \mathrm{~s} / \mathrm{mm}^{2}$.

\subsection{Structural MRI imaging protocol}

T1w MPRAGE 3T scans were acquired with $\mathrm{TR}=2400 \mathrm{~ms}$, TE= 2.14, $\mathrm{TI}=1000 \mathrm{~ms}$ and flip angle of $8^{\circ}$, isotropic $0.7 \mathrm{~mm}$ voxels and FOV $224 \mathrm{~mm} \times 224 \mathrm{~mm}$. Part of the HCP minimal processing pipeline aligns these images to the diffusion data and re-slices them to $1.25 \mathrm{~mm}$ isotropic resolution. These were visually checked prior to processing to ensure good alignment between $\mathrm{T} 1 \mathrm{w}$ and diffusion data.

\subsection{MRI pre-processing}

Diffusion data pre-processing was performed using FMRIB Software Library (FSL) v5.0 tools. Diffusion tensor model fitting was done with DTIfit; Bedpostx GPU (Hernández et al., 2013) was used to estimate three fibers per voxel keeping the remaining FSL parameters at their default settings.

The structural data pre-processing pipeline used the $1.25 \mathrm{~mm}$ isotropic T1w data in diffusion space. SPM12 (https://www.fil.ion.ucl. ac.uk/spm/software/spm12/), running in MATLAB R2017a, was used. Segment (Ashburner and Friston, 2005) segmented the T1w images into CSF, grey matter (GM) and white matter (WM). The Shoot diffeomorphic registration algorithm (Ashburner and Friston, 2011) used these segmentations to create a group average space with which each individual was aligned. Total intracranial volumes were calculated using the 
Tissue Volumes function. Skull-stripped T1w images were obtained using a mask created from the sum of the GM, WM and CSF segmentations, which was thresholded at 0.1 . For quality control, the following results were visually assessed for every subject: A. The SPM generated segmentations (GM, WM, CSF), B. The resulting templates from SPM shoot, C. The resulting warped $\mathrm{T} 1 \mathrm{w}$ data in group average space. These steps ensured the alignment, warping and any analysis in group average space were as accurate as possible.

\subsection{Tractography targets}

We replicate the method of Akram et al (2018). For this study, the following bilateral regions had already been defined on MNI ICBM 152 non-linear (6th Generation) symmetric standard-space T1-weighted average structural template image ( $1 \mathrm{~mm}$ resolution): primary motor cortex (M1), thalamus, dentate nucleus. As the DTCp is a decussating fibre pathway, contralateral cerebrum and ipsilateral cerebellum canonical exclusion masks were also used. To accurately estimate warps between the HCP population average space and MNI space, warps between the group average T1w images were estimated using FSL FNIRT, and the results visualised to ensure good alignment. All of the canonical seed, target and exclusion masks were then warped to the group average HCP space. These binary images were then warped, using nearest neighbour interpolation, to the individual subject space using the deformation field generated via Shoot.

\subsection{Probabilistic tractography}

FSL's probabilistic tractography tool for NVIDIA GPUs, Probtrackx GPU (Hernandez-Fernandez et al., 2019), was used. Every voxel was sampled 5000 times, using the three fiber distributions option, with a curvature threshold of 0.2 . The proportion of samples that depart from a seed voxel and reach a target ROI is defined as the probabilistic index of connectivity (PICo). In this study, PICo ranged from 0 to 0.5 and was used to construct a sparse connectivity matrix. To avoid tracking into the ventricles, subarachnoid space and the remainder of CSF spaces, a threshold of 0.2 was used to binarize the CSF segmentation, which was employed as a termination mask. All tractography used the canonical exclusion masks described above. As per Akram et al (2018), the following tractography strategy was used to reconstruct the DTCp:

- Seed: M1; Waypoints: Contralateral cerebellum and ipsilateral thalamus.

- Seed: Cerebellum; Waypoints: Contralateral M1 and thalamus.

No other termination rules (e.g. masks, regions of interests, voxel thresholds) were used. To reconstruct the DTCp, both outputs from tractography (fdt_paths file from each probtrackX result) were summed and divided by the maximum PICo value within the resulting image, to normalise the range to between $0-1$. These probabilistic DTCp reconstructions were then warped to group average space using the deformation field generated via Shoot and trilinear interpolation. For visualisation purposes, using "imcalc" in SPM12 all warped DTCp on each side were used to calculate the average DTCp for each hemisphere (Fig. 2).

\subsection{Tractography-defined Vim localisation}

The Vim region was defined as the intersection of the DTCp with the respective thalamic mask for each subject. Probabilistic tractography, despite better resolving grey-white matter transitions and complex fibre architectures, typically yields more false positive tracts; a threshold must be applied to improve specificity, while inevitably reducing sensitivity (Zalesky et al., 2016). While thresholding is a necessity, there is no consensus on the ideal limit and the choice of threshold remains arbitrary (Li et al., 2012). Given the potential variation in connectivity strength with tracking distance and seed volume, both of which may correlate with head size, and that fixed PICo thresholds may bias centroids towards regions with higher anisotropy (i.e. areas likely to have higher PICo values), we opted to use an adaptive threshold for the DTCpthalamus intersection images by binarizing at $20 \%$ maximum connectivity (i.e. PICo) within the intersection region.

The volume of each intersection ROI was calculated in $\mathrm{mm}^{3}$. Each ROI was then warped to the HCP group average space, and bwconn in MATLAB used to ascertain the coordinates of each individual centroid in group average space. All reported coordinates in this work have been described in the anterior-posterior (AP), medio-lateral (ML) and superior-inferior (SI) directions, following standard stereotactic convention. Voxel-to-world mapping has been used for all reported results.

The average centroid locations were calculated for each side. To compare each individual centroid to the group average coordinates, four displacement metrics were calculated for each ROI: These were the distance (absolute displacement) between the individual and average coordinate in each axis (medio-lateral, anterior-posterior and superiorinferior) and overall Euclidean distance (ED), using voxel-to-world mapping to provide results in $\mathrm{mm}$. To compare differences between average world-locations of centroids between hemispheres, a two-sample repeated measure $t$-test was used (using the absolute values in the ML axis). To compare differences in variability between sides, a PitmanMorgan test was used (Gardner, 2001).

Results $P<0.05$ were deemed to be significant. Centroid locations were summarised using the root mean square of the Euclidean distance, maximum Euclidean distance, and maximum absolute displacement in each axis.

\subsection{Comparison to the surgical standard}

As the Vim is not visible on conventional stereotactic MRI, surgery is generally performed through atlas-based coordinate targeting, which consists in applying to each patient a widely used transformation matrix for an atlas defined target (Alho, 2011; Melo, 2021)

To assess the accuracy of this approach, we calculated the position of the Vim on the HCP group-average template using atlas-based coordinates. These are defined in relation to the mid-commissural point (MCP) of the anterior commissure-posterior commissure (AC-PC) plane, in relation to the following formula (Akram et al., 2018):

- AP direction = (AC-PC length $) / 3-2 \mathrm{~mm}$ anterior to PC

- ML direction = Midline \pm 12 to $14 \mathrm{~mm}$; (12.5 mm used in this study)

- SI direction $=0 \mathrm{~mm}$

We projected this location onto the group average thalamus along with the all tractography calculated centroids using the render toolbox in SPM12. We also calculated the absolute displacement of the tractography centroids from the atlas-based targets as outlined above and tested for differences along each axis using a one-sample $t$-test. Moreover, surgical trajectory planning to the atlas-based Vim coordinates was carried out on both sides by an experienced functional neurosurgeon (HA) on HCP group average template using a standard surgical approach and the BrainLab Elements planning platform. In short, entry points were planned on or adjacent to the coronal suture with a lateral angle that allows for trajectories that avoid sulci and the lateral ventricles, maximise the intrathalamic course, and avoid close proximity to the internal capsule.

\subsection{Investigation of confounds}

Methods developed to augment neurosurgical targeting will only be translational if they are robust to artifacts and reflect only true anatomical variability, to ensure safety and efficacy in their application. While HCP data has been pre-processed to correct for motion artefacts, the fact that DWI is extremely sensitive to movement (Baum et al., 2018; Le Bihan et al., 2006; Ling et al., 2012; Muller et al., 2020; Tijssen et al., 

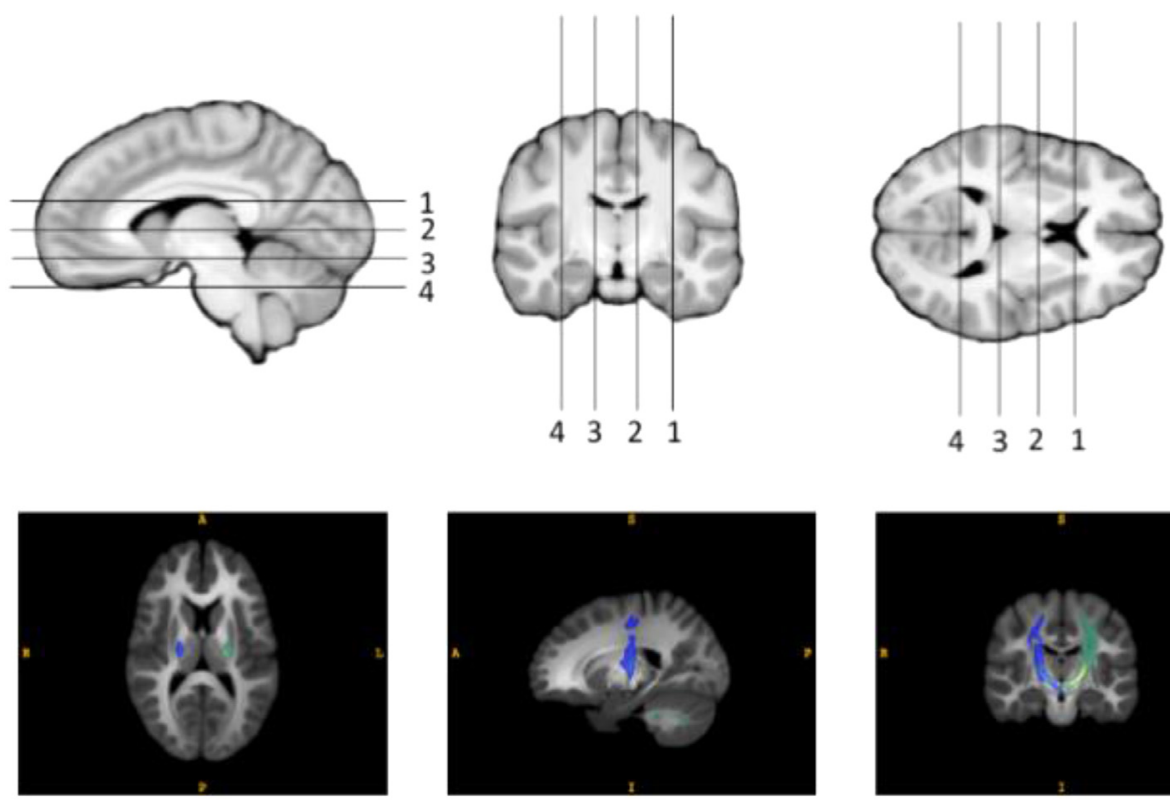

Fig. 2. Right (blue) and left (green) group average DTCp windowed between PICo $0-1$. Values $<0.05$ were zeroed for visualisation. (For interpretation of the references to color in this figure legend, the reader is referred to the web version of this article.)

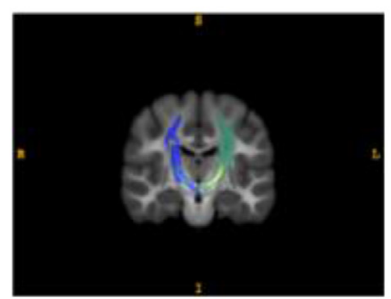

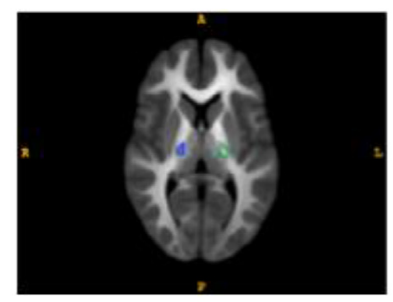
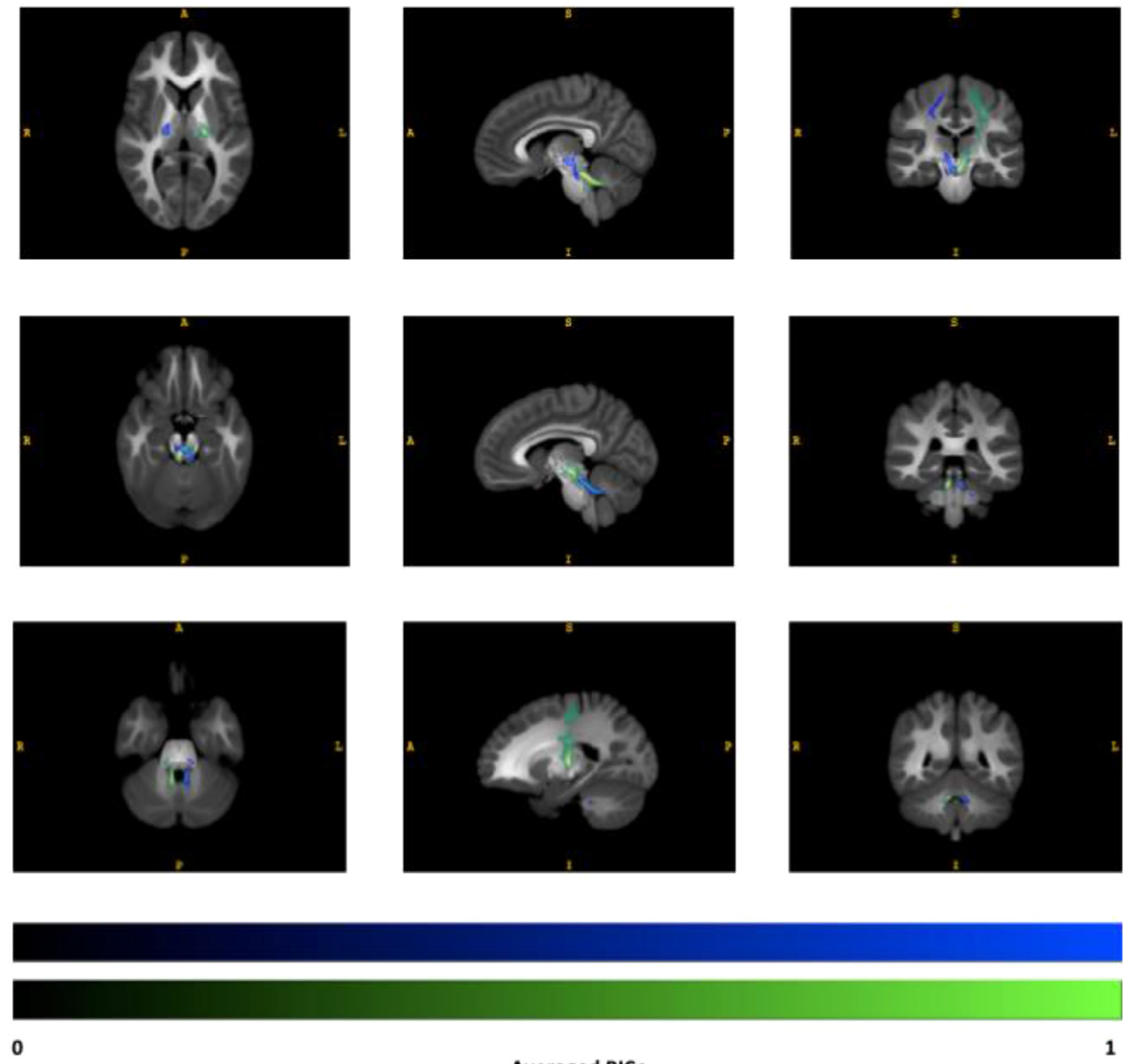

Averaged PICo

2009) drove efforts in this work to establish the influence of subject movement on eventual observed Vim variability.

\subsubsection{Methodology confounds}

Participant motion was tested using the output from FSL "eddy", contained in the eddy_movement_rms file, which consists of two measures of total movement: the root mean square relative to the first volume acquired, and the immediate preceding volume through the acquisition.

Beyond motion artefacts, the choices of seed and target region can heavily influence the resultant structural connectivity obtained by tractography (Ambrosen et al., 2020). Specifically, tracking target volumes may influence the probability of connectivity, so we tested the influence of the volumes of the M1 and cerebellar masks. The probability of con- 
nectivity falls the further from the seed a streamline passes; hence we used total intracranial volumes (TIV) as a surrogate measure of tracking distance across the population.

For each hemisphere, we tested a total of five confound measures of interest (two movement, two ROI volume, TIV) against each of the fourdisplacement metrics (displacement in anterior-posterior, medio-lateral and superior-inferior directions and Euclidean distance). An F test was used to test each confound against the centroid coordinates for each hemisphere. We performed Bonferroni statistical correction $\mathrm{P}<0.007$ for multiple comparisons.

\subsubsection{Superior cerebellar peduncle as an anatomical confound}

Based on the known anatomy of the DTCp and the inherent limitations of probabilistic tractography, we identified the superior cerebellar peduncle (SCP) as a potential site that may critically influence our model. This is because the SCP is a narrow white matter structure, 2$4 \mathrm{~mm}$ in width in healthy adults and less in certain disease populations (Tsuboi et al., 2003), which is surrounded by CSF. In the HCP protocol, it is represented by 2-4 voxels and is the narrowest structure the DTCp fibres must cross. We hypothesised that SCP size may positively correlate with the connectivity metrics in the resulting tracts. For this, we manually segmented the left and right SCP on the group average template using ITK-SNAP. For each individual, we integrated the Jacobian determinant values in these regions to provide a volumetric measure. We then extracted the maximum PICo value for each DTCp and correlated this against the corresponding SCP volume (left DTCp vs right SCP and vice versa).

\subsection{Test-retest reliability}

Our goal in this work was to demonstrate inter-subject anatomical variability through the use of probabilistic tractography. To address the possibility that the use of dMRI and the chosen processing pipeline could have introduced noise, eventually erroneously interpreted as variability, we used HCP test-retest dataset to assess the reproducibility of our results in the same individual. The test-retest dataset is a subset of the 1200 HCP individual MRIs (Van Essen et al., 2013) consisting of 45 individuals cohort (13 male, 22-35 years old) obtained over variable intervals $(4.7 \pm 2$ months interval). Identical HCP minimal processing steps have been applied. We applied the same Vim processing methods as outlined above for the S100 dataset, to 44 individuals from the HCP test-retest cohort (we found one individual had no test-retest DWI data). We then compared the displacements (AP, ML, SI, ED) between the paired, within subject, cd-Vim centroids (i.e., scan1 - scan2) to the first scanning session displacements of all individuals to the average centroid (i.e., scan 1 - average) using a Wilcoxon sign rank test (left tail to reflect a priori expectation that test-retest results will be smaller), again taking any results $\mathrm{P}<0.05$ as significant.

\section{Results}

\subsection{Demographics}

The cohort consisted of young adults, ranging from 22-33y with an average age of $29.11 y$ ( $\pm 3.67 y, 54 \%$ female) and average brain volume $1.42 \mathrm{~L}( \pm 0.14 \mathrm{~L})$.

\subsection{DTCp tract reconstruction}

Fig. 2 shows the group average reconstructed right and left DTCp, showing the tracks passing from the primary motor cortex, with the majority decussating in the brachium conjunctivum to the contralateral superior cerebellar peduncle and cerebellum. A secondary tract bundle, decussating in the dorsal mid-pons to the contralateral middle cerebellar peduncle and cerebellum is noted, and corresponds to the corticopontocerebellar pathway (Palesi et al., 2017). Note, Fig. 2 has been rescaled so
Table 1

Average (SD) tractography Vim location (world coordinates). Positions posterior to the anterior commissure, left of the mid commissural plane and inferior to axial AC-PC plane are denoted by negative values.

\begin{tabular}{llll}
\hline & \multicolumn{2}{l}{$\begin{array}{l}\text { Average tractography centroid world coordinates } \\
\mathbf{m m} \pm \text { standard deviation (SD) }\end{array}$} \\
\cline { 2 - 4 } & $\begin{array}{l}\text { Anterior- } \\
\text { posterior } \\
(\text { AP) }\end{array}$ & $\begin{array}{l}\text { Medio- } \\
\text { lateral } \\
(\mathbf{M L})\end{array}$ & $\begin{array}{l}\text { Superior- } \\
\text { inferior } \\
(\mathrm{SI})\end{array}$ \\
\hline Left Vim & $-18.78( \pm 1.14)$ & $-13.71( \pm 1.22)$ & $2.67( \pm 1.33)$ \\
Right Vim & $-17.61( \pm 1.49)$ & $13.09( \pm 1.15)$ & $3.94( \pm 1.60)$ \\
\hline
\end{tabular}

that the group average PICo values range between 0 and 1 (left rescaling $\mathrm{PICo}=0.5758$, right rescaling PICo $=0.5227$ ), as the physical properties of the DRTC reconstruction method used would naturally result in lower average PICo values (i.e. capturing the mid-point intersection between two long-range tracking results from seed voxels situated far apart from one another). The population percentage overlap, thresholding each individual track by $20 \%$ maximum PICo within the tract-thalamus intersection region, has been provided in the supplementary material (supplementary figure 1) to show high levels of group overlap in the region of the Vim, which was the objective of the tracking procedure. Probabilistic DTCp reconstructions for five subjects (supplementary figures 2-6) have also been provided in the supplementary material, showing the method is reproducible across individuals."

\subsection{Tractography-defined Vim}

Table 1 summarises tractography-defined left and right Vim coordinates. Overall, the left Vim was located significantly more posterior, inferior and lateral compared to the right $(\mathrm{P}<0.00001)$. There was more variability in Vim location on the right in the AP and SI axes $(\mathrm{P}=0.02$ and $\mathrm{P}=0.03$ respectively). Table 2 summarises the dispersion metrics for the tractography defined centroids compared to the group average. Fig. 3 shows the principal directions of variability. The median Vim volume was $110.35 \mathrm{~mm}^{3}$ on the left and $168.71 \mathrm{~mm}^{3}$ on the right, which was significantly different between hemispheres $(P=0.01)$.

\subsection{Vim coordinates: Atlas-based coordinates versus connectivity-derived coordinates}

Table 3 summarises the dispersion metrics for the connectivitydefined centroids compared to the atlas-based surgical coordinates. These were significantly different in all axes, with the surgical coordinates located more medial and inferiorly. The difference was least in the AP plane, located more posteriorly on the right and anteriorly on the left (Fig. 4). Fig. 5 projects this data onto the thalamus.

\subsection{Methodological confounds}

No significant effects were found for any of the possible methodological confounds (eddy movement parameters, M1 or cerebellar target mask volume, TIV), with statistical significance Bonferroni correction $\mathrm{P}<0.007$ for multiple comparisons.

\subsection{Superior cerebellar peduncle}

No difference between left and right SCP volumes was found. On the left (i.e. left DTCp vs right SCP), SCP volumes significantly correlate with PICo max, mean and variability. On the right, no correlation exists.

\subsection{Test-retest reliability}

Test-retest cd-Vim positions showed between subject displacement were significantly greater than within subject displacement (left root 
Table 2

Dispersion metrics for tractography-defined Vim centroids (mm).

\begin{tabular}{llllll}
\hline & $\begin{array}{l}\text { Root mean square Euclidean } \\
\text { distance }(\mathrm{ED}) \text { from average } \\
\text { tractography centroid }(\mathrm{mm})\end{array}$ & $\begin{array}{l}\text { Maximum ED from average } \\
\text { tractography centroid } \\
(\mathrm{mm})\end{array}$ & \multicolumn{4}{l}{$\begin{array}{l}\text { Maximum absolute distance from average tractography centroid } \\
(\mathbf{m m})\end{array}$} & Anterior-posterior & Medio-lateral & Superior-inferior \\
\cline { 4 - 7 } & & 4.38 & 2.85 & 3.29 & 3.59 \\
Left Vim & 2.14 & 6.74 & 5.99 & 3.13 & 4.16 \\
Right Vim & 2.47 & & & & \\
\hline
\end{tabular}

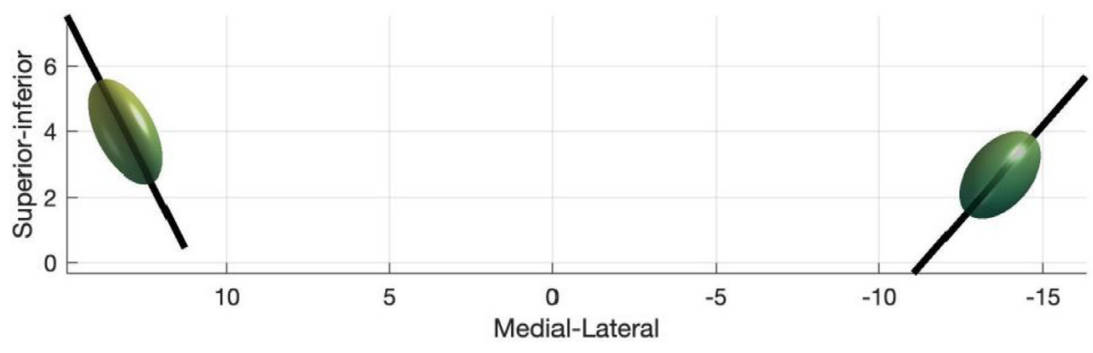

Fig. 3. Principal directions of variance for tractography-defined Vim. The eigenvector corresponding to the first principal component is shown as solid line.

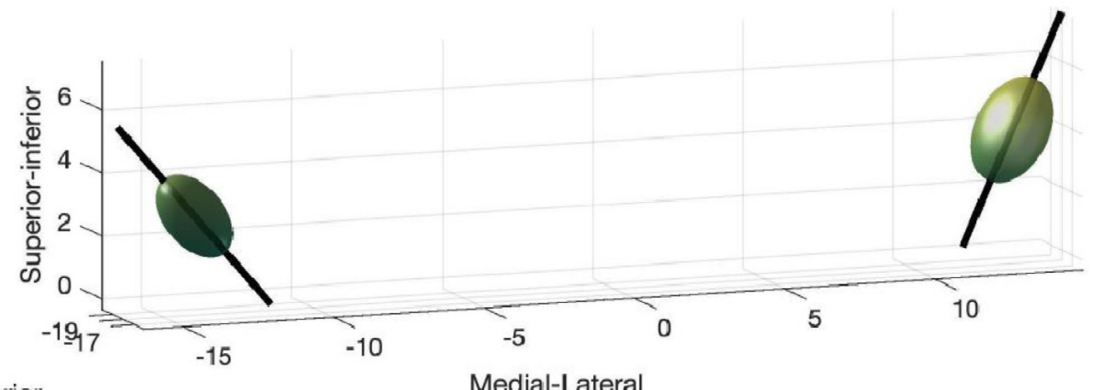

Anterior-posterior

Medial-Lateral

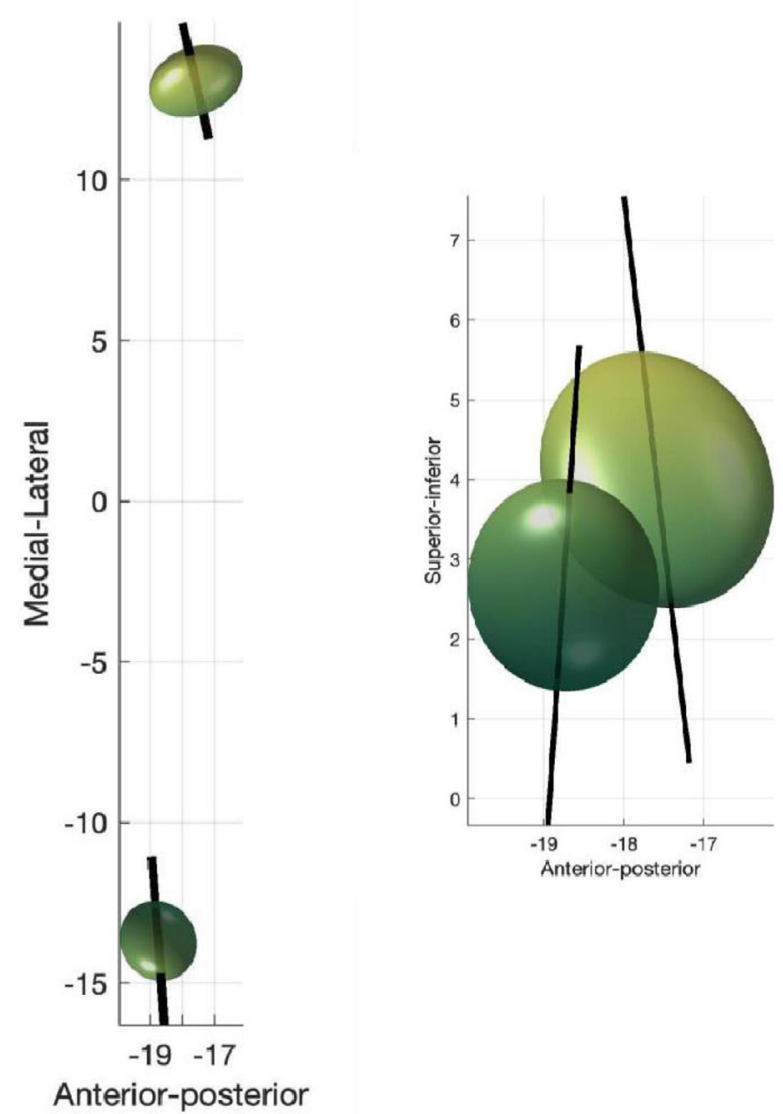


Table 3

Distances from atlas-defined coordinates.

\begin{tabular}{llllll}
\hline & $\begin{array}{l}\text { Root mean square Euclidean } \\
\text { distance (ED) from atlas-defined } \\
\text { coordinates (mm) }\end{array}$ & $\begin{array}{l}\text { Maximum ED from } \\
\text { atlas-defined } \\
\text { coordinates }(\mathbf{m m})\end{array}$ & & \multicolumn{2}{c}{ Maximum absolute distance from atlas-defined coordinates (mm) } \\
\cline { 3 - 6 } & & 7.26 & Anterior-posterior & Medio-lateral & Superior-inferior \\
\hline Left Vim & 4.24 & 9.92 & 2.73 & 4.57 & 7.01 \\
Right Vim & 5.71 & 6.87 & 4.54 & 8.86 \\
\hline
\end{tabular}

Table 4

Summary of test-retest cd-Vim positions using 44 paired datasets from the Human Connectome Project.

\begin{tabular}{llll}
\hline & \multicolumn{2}{l}{ Average tractography centroid world coordinates mm \pm standard deviation (SD) } \\
\cline { 2 - 4 } & Anterior-posterior & Medio-lateral & Superior-inferior \\
\hline Left Vim: Session 1 & $-18.02( \pm 1.35)$ & $-13.35( \pm 1.45)$ & $1.09( \pm 1.78)$ \\
Left Vim: Session 2 & $-18.02( \pm 1.24)$ & $-13.22( \pm 1.36)$ & $0.98( \pm 1.49)$ \\
Right Vim: Session 1 & $-17.15( \pm 1.54)$ & $12.45( \pm 1.25)$ & $2.22( \pm 2.12)$ \\
Right Vim: Session 2 & $-17.16( \pm 1.34)$ & $12.56( \pm 1.10)$ & $2.23( \pm 1.80)$ \\
\hline
\end{tabular}

mean square Euclidean distance within $1.83 \mathrm{~mm}$, between $2.72 \mathrm{~mm}$; right root mean square Euclidean distance within $2.13 \mathrm{~mm}$, between $2.96 \mathrm{~mm}, \mathrm{P}<0.005$ ), and each displacement axis (AP, ML, SI) was also significant, $\mathrm{P}<0.05)$. The average positions are shown in Table 4:

\section{Discussion}

A connectivity-based approach was used to characterize the intersubject variability of the DTCp in 100 unrelated subjects from the WUMinn HCP dataset. The DTCp was chosen as it is part of the tremor network that can be targeted in functional neurosurgery in the treatment of severe refractory tremor disorders, such as PD and ET (Anderson et al., 2011). The Vim, not visible on conventional MRI, occupies a central position in the DTCp. The inability to directly visualise the Vim on conventional imaging, and the consequent reliance on atlas-based coordinates for surgical planning, may lead to suboptimal targeting and poor long-term clinical outcomes (Akram et al., 2018). While demonstrating Vim inter-subject variability and discrepancy from atlas-based Vim coordinates, we also confirmed reproducibility by using the test-retest HCP cohort. We aimed to exclude a number of possible methodological confounds, including tracking target volume, head size and subject movement in the scanner. None were found to be statistically significant after Bonferroni correction $\mathrm{P}<0.007$ for multiple comparisons. We found that the SCP volume influenced PICo on the left, and therefore may represent a bottleneck for probabilistic tractography; a pontine target, prior to entering this structure, may help generate more consistent results. There were hemispheric asymmetries in the probabilistic DTCp reconstructions, which may be accounted for by intrinsic anatomical idiosyncrasies of lateralized cerebral and cerebellar connectivity.

\subsection{Vim localisation}

Ex vivo, it is known that the precise location of Vim varies considerably among subjects (Morel et al., 1997). This represents a significant challenge for the surgical treatment of tremor, where accurately locating Vim in individual subjects is considered to be key for improving therapeutic outcomes (Akram et al., 2018; Pouratian et al., 2011; Tsolaki et al., 2017). Indeed, in Vim DBS it has been shown that an error in lead placement of just $2 \mathrm{~mm}$ can lead to a significant impact on efficacy (Papavassiliou et al., 2004). Atlas-based targeting is defined as procedures where the location of a brain structure coordinates is defined by transforming some kind of canonical map(s), atlas or coordinates to individual subject space often using standard MRI sequences (i.e. the coordinates are primarily based on the atlas alone). These atlas-based methods remain the standard practice for Vim targeting in functional neurosurgery, and are widely recognised to be sub- optimal according to both functional and structural connectivity studies (King et al., 2017; Nowinski et al., 2005), confirming the discrepancies between atlas-based coordinates and individual Vim localization (Akram et al., 2018). Direct approaches, to visualise a structure at an individual subject by leveraging intrinsic MR biophysical properties, either via bespoke sequences or novel metrics, that may also use of some form of prior (e.g. atlas, probability map) to support, or augment, this primarily individual level direct mapping, offer new ways to better capture individual variability. In this context, probabilistic tractography represents a promising methodology to demarcate the internal structure of the thalamus at an individual subject level in vivo (Akram et al., 2018; Behrens et al., 2003; Middlebrooks et al., 2018b; Pouratian et al., 2011; Traynor et al., 2010). Exploiting the anatomical properties of the DTCp, it is possible to reconstruct this tract using probabilistic tractography to help identify the Vim in vivo (Palesi et al., 2015). Motivated by the translational potential of these methods, we used the method proposed by Akram et al (2018) to better characterise variability of tractographydefined Vim location and determine whether there were any methodological confounds that may influence these results. In the HCP data we found that subject motion, ROI volumes and TIV did not significantly influence results. In line with earlier work (Akram et al., 2018; Middlebrooks et al., 2018b; Papavassiliou et al., 2004; Tsolaki et al., 2017), we demonstrated a significant disparity between tractographydetermined and the atlas-determined Vim coordinates. Furthermore, we found that variability in the tractography defined Vim locations was asymmetric, more marked on the right, and that the variation mainly occurred along the anterior-posterior and superior-inferior axes. We also demonstrated that tractography defined Vim locations tended to be located posterio-medially when compared to a standard surgical trajectory. These errors, orthogonal to the standard electrode trajectory (Fig. 5), are likely to have the greatest clinical impact. Knowledge of the displacement between atlas and tractography defined Vim may help augment surgical planning in the future, either by using the information to modify surgical trajectories or where this is not possible, for example due to the proximity of eloquent anatomical structures, through the use of directional DBS electrodes.

\subsection{Cerebellar tract asymmetry}

This work found evidence of hemispheric differences in Vim position (both the average position and the positional variability), and also in the relationship between DTCp connectivity metrics and the SCP. Our method to construct Vim centroids is reliant on estimating DTCp via probabilistic tractography, and therefore these results reflect hemispheric asymmetries in the reconstructed tract. Whilst cerebellar functional asymmetry is well established, to our knowledge cd-Vim intrasub- 


\section{Left Vim: AP}

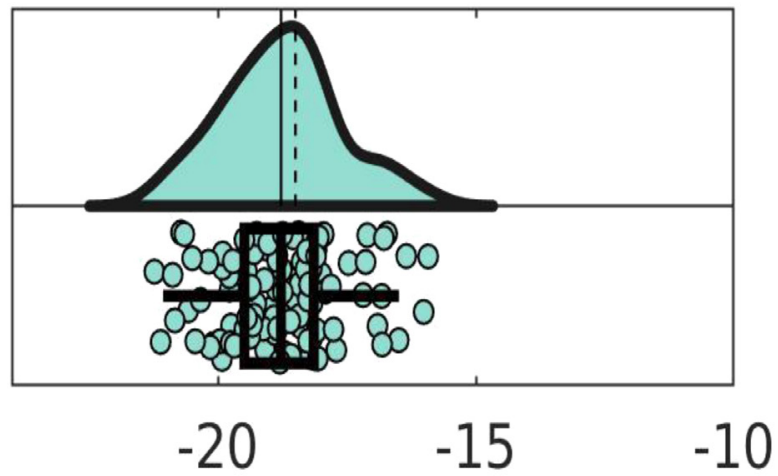

Left Vim: ML

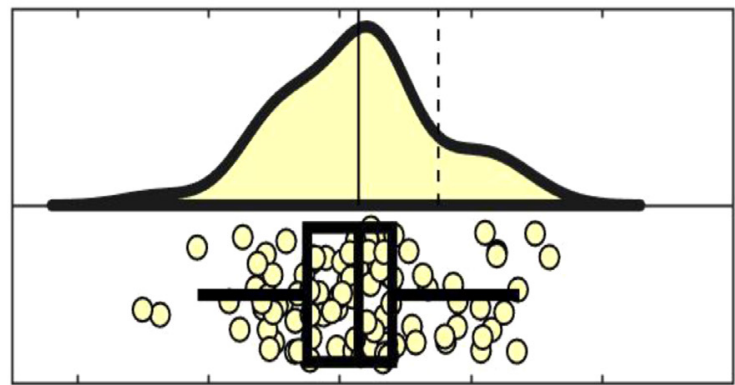

$\begin{array}{llllll}-18 & -16 & -14 & -12 & -10 & -8\end{array}$

Left Vim: SI

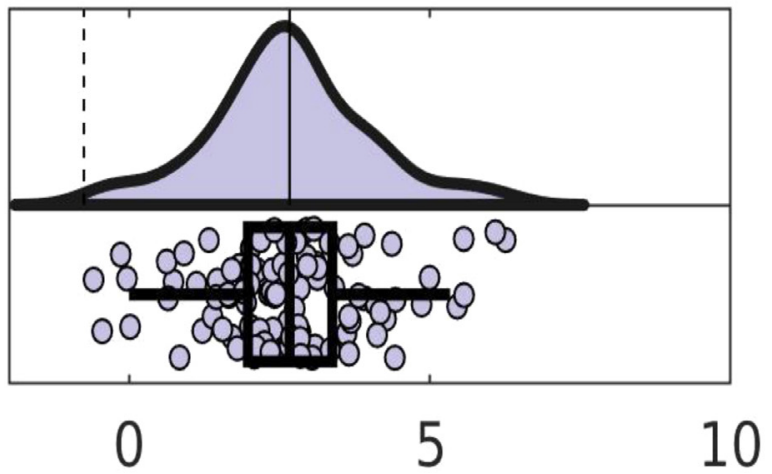

Right Vim: AP

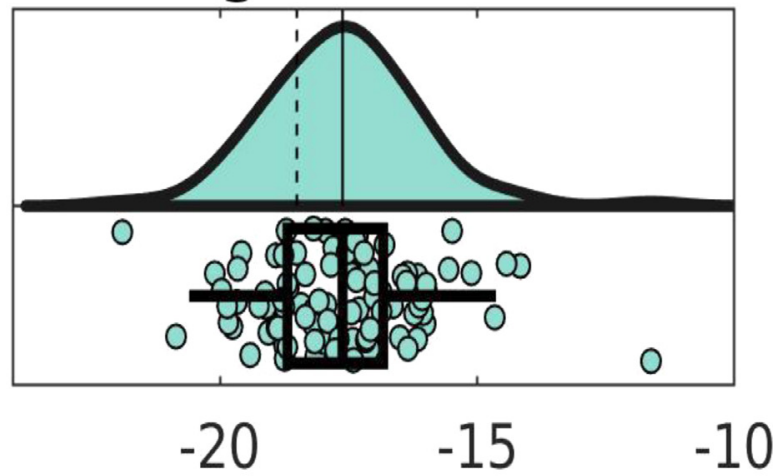

Right Vim: ML

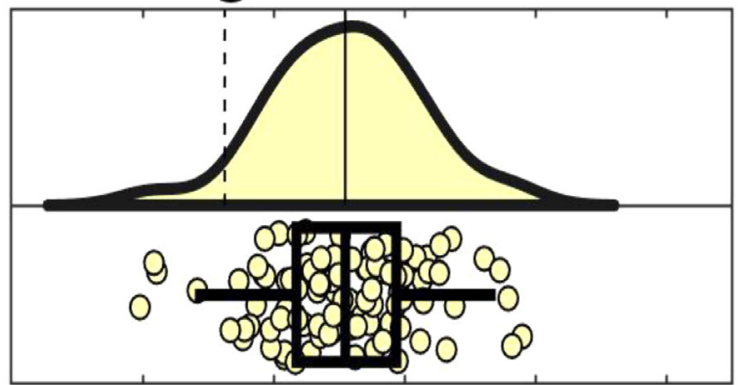

$\begin{array}{llllll}8 & 10 & 12 & 14 & 16 & 18\end{array}$

Right Vim: SI

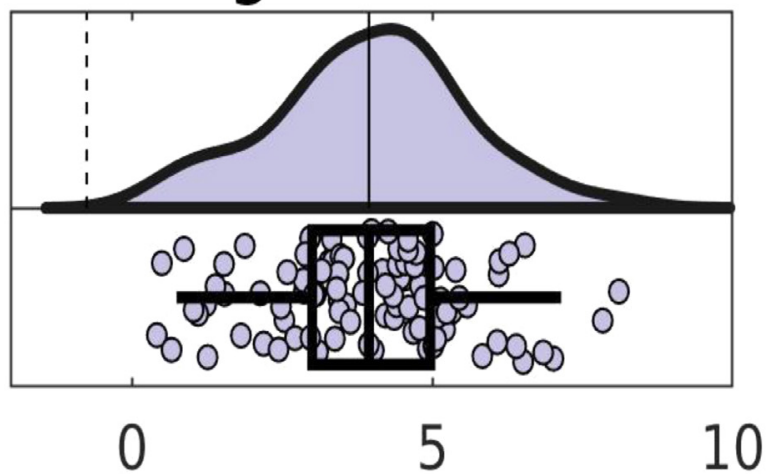

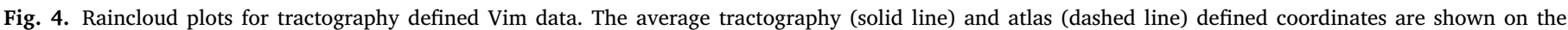

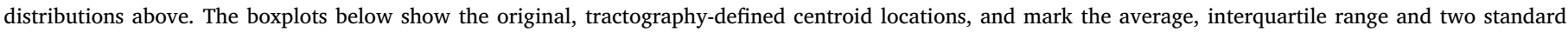
deviations for each axis (AP, ML and SI), in mm.

ject asymmetry has not been previously reported. It is well established that the cerebellum is involved in a wide range of functions, including behaviour, cognition and language (Bostan et al., 2013; Strick et al., 2009). In some of these non-motor activities, marked left-right asymmetries have been demonstrated on fMRI (King et al., 2019) that mirror those seen in the association cerebral cortex, particularly for language generation (Wang et al., 2013). Therefore, one possible explanation for some of the observed DTCp asymmetry may relate to its role in language. Certainly, damage to the right cerebellum has been reported to cause non-fluent aphasias and agrammatic speech. We used the metaanalysis tool NeuroSynth (http://neurosynth.org) to identify language fMRI studies using the search term "words". This calculated an activation map based on pooled results from 944 studies and revealed a region of significant activation in the inferior portion of the primary motor cortex that fell within the M1 seed mask used in this work (Fig 4). Therefore, some of the asymmetries noted in this work may simply be due to the M1 mask including this speech region (Fig 6).

\subsection{Limitations}

There are a number of potential limitations in this work. There is no gold standard or ground truth for comparison and validation of the tracts generated. However, our objective was to quantify the observed variance and try to exclude possible methodological confounds, as a necessary foundation for developing future translational tools. While the cd-Vim centroids of some individuals in this work seemed to be quite displaced from the atlas-based surgical coordinate, in the absence of clinical outcomes it is not possible to weigh up which solution is more 
A.
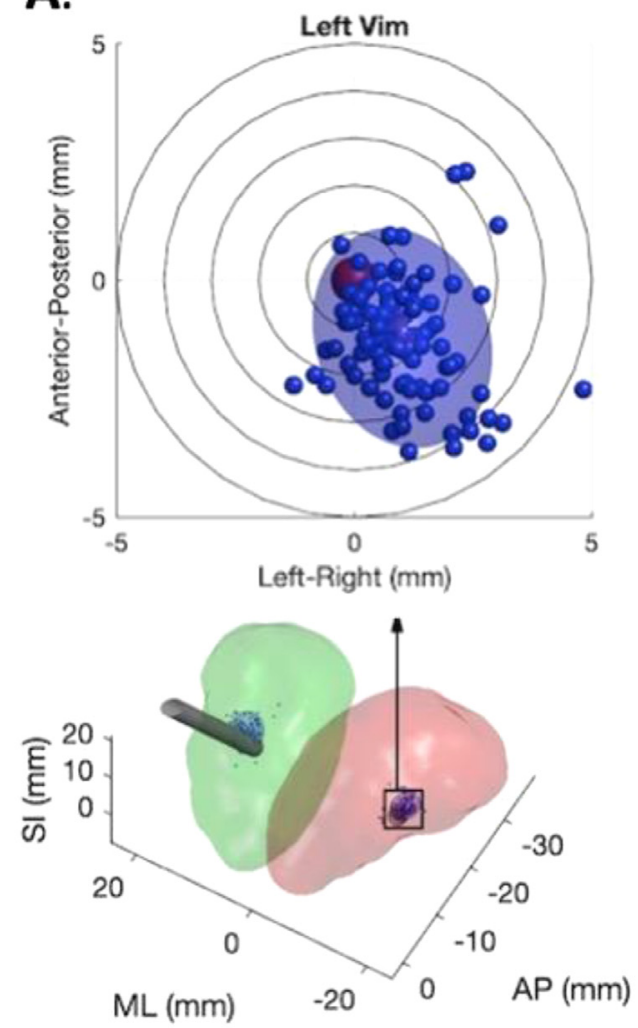

B.
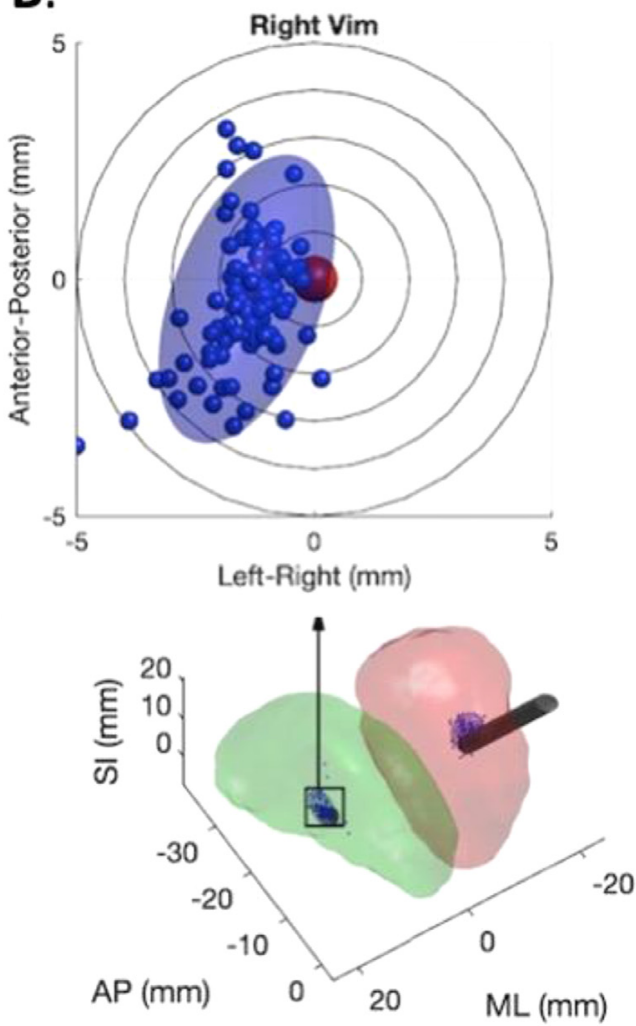

Fig. 5. DBS trajectory planning using atlas-based coordinates. View down bore of left (A) and right (B) thalamic electrode, with individual tract centroids and variance projected orthogonal to the electrode trajectories; Coronal (C) and Axial (D) views of DBS electrode trajectory showing thalamic renderings (top) and original planning MRI (bottom).

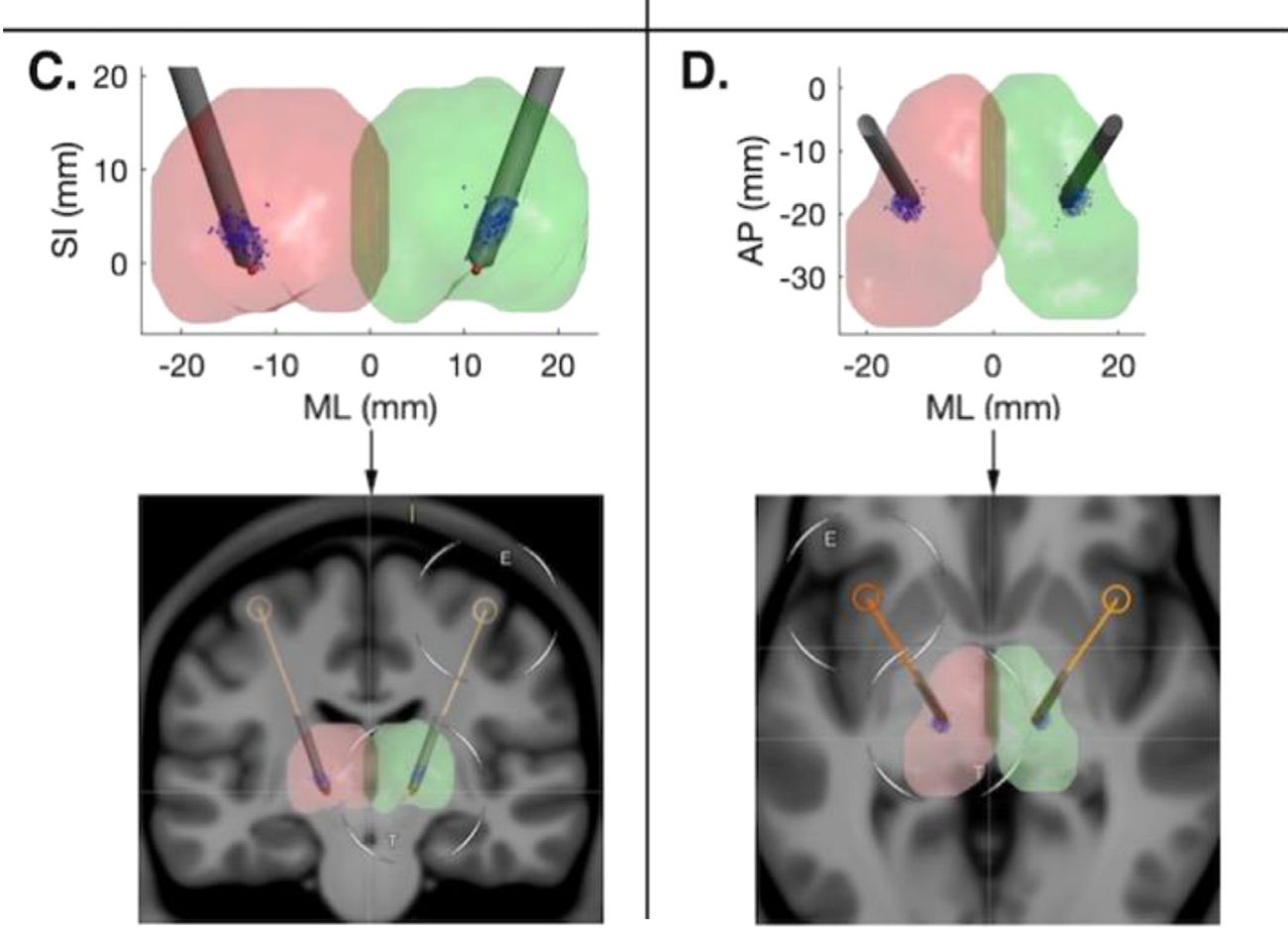

correct. In future work, we will apply these methods to clinical data paired with DBS outcomes, where these questions can be answered and other techniques to refine the pre-implantation accuracy explored. Furthermore, it is possible that histological ground truth does not necessarily need to perfectly match when the aim is to generate targeting maps to optimise functional surgical outcomes. The population used in this work, consisting of young healthy adults, may not accurately represent patients who require surgery for tremor, and it is possible that methodological confounds examined here may have a greater impact in clinical cohorts with movement disorders, necessitating additional steps to control for errors. Furthermore, some measure of ground-truth is required to align any imaging approach with the underlying functional anatomy, as validation. An example of this is the fact that while the cd-Vim centroids of some individuals in this work seemed to be quite displaced from the 

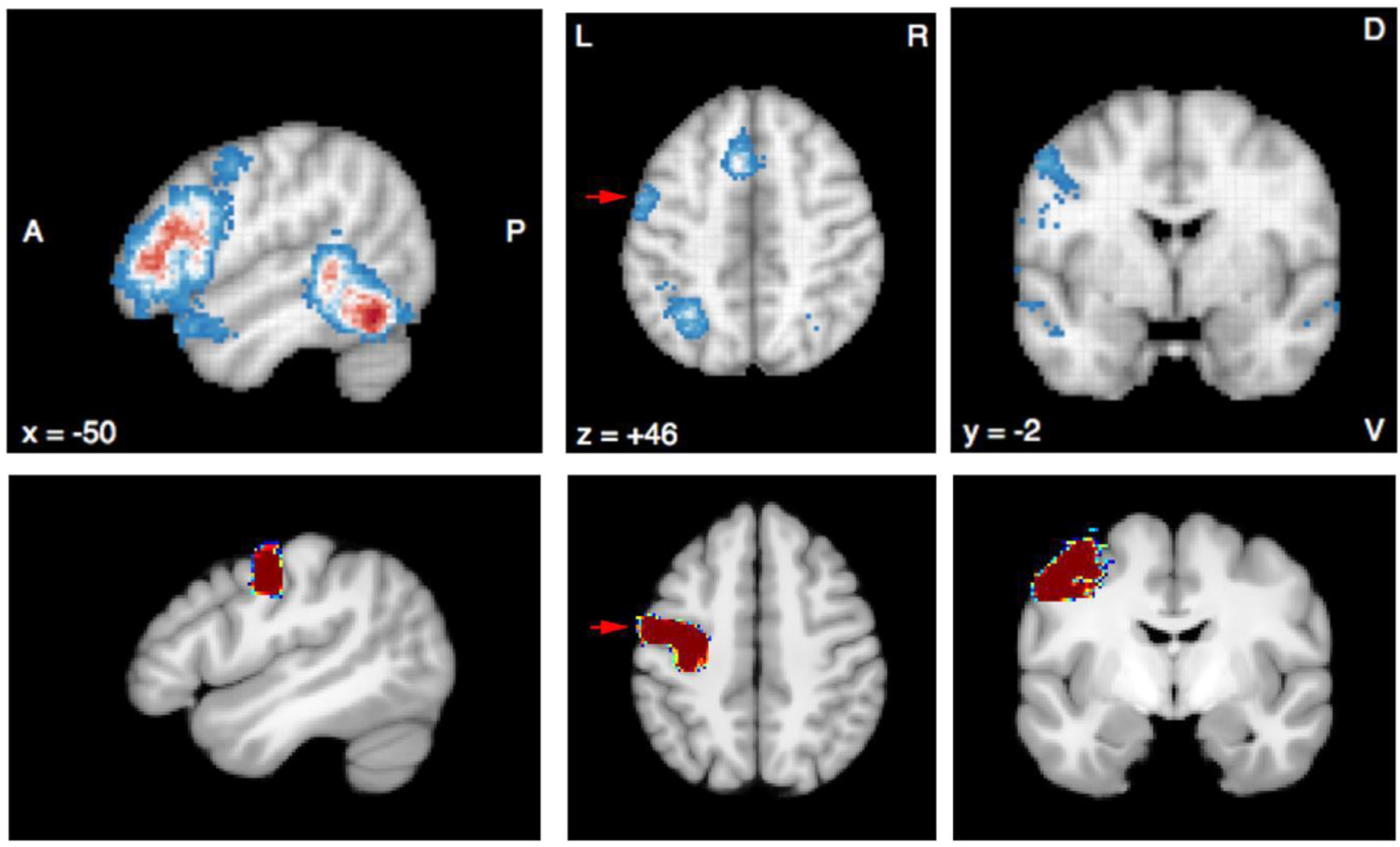

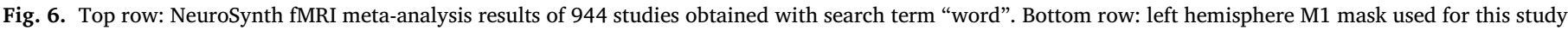
(arrow).

atlas-based surgical coordinate, in the absence of clinical outcomes it is not possible to weigh up which solution is more correct. In future work, we will apply these methods to clinical data paired with DBS outcomes, where these questions can be answered and other techniques to refine the pre-implantation accuracy explored. While the method developed appears to be robust, additional steps may be needed to achieve comparable results in clinical populations; this could include the acquisition of DWI following general anaesthesia to minimise any effect of movement.

Our work used discrete a priori anatomical constraints to reconstruct a single well-characterised tract of interest (Akram et al., 2018). Whilst this approach may reduce issues associated with complex-fibre populations and false positive tracts (Maier-Hein et al., 2017), some of the steps, such as choice of target ROIs, masking errors or tract thresholding, may represent additional sources of error influencing the reconstructed anatomy. In probabilistic tractography, thresholding is applied in an attempt to minimise spurious connections, which impact the reconstructed tracts more negatively than false negatives (Zalesky et al., 2016), and the more stringent a threshold the greater the effect in reducing network density (Li et al., 2012). Furthermore, if subjects have different sparsity in their connectivity matrices, thresholding will cause further variations on network metrics, affecting short-term reproducibility of structural connectivity (Tsai, 2018). A potential way to control for this is the application of several connectivity thresholds to characterise the obtained networks (Buchanan et al., 2020). If the sparsity is different within subjects, integration over different connectivity thresholds would represent a way to estimate network metrics relatively reliably (Tsai, 2018). Alternatively, threshold-free approaches have been proposed (Lambert et al., 2017) which avoid this step and may provide more stability.

We found that SCP volume positively correlates with PICo values, an effect that may be accentuated in specific clinical populations. Therefore, choosing more proximal targets, such as the ponto-SCP junction, may eliminate this problem and lead to more reliable results. Finally, there is also a risk that some of the variance could emerge simply through image registration errors, which in this work was primarily achieved using T1w data. Given this, possible approaches to address these issues in the future would be to either adopt non-linear registration models designed for DWI data (Chen et al., 2019; Zhang et al., 2006), or use complementary thalamic segmentation methods to improve the alignment of the internal nuclei (Iglesias et al., 2018; Lambert et al., 2017).

Finally, there are a number of emerging optimised MRI sequences to help target DBS structures, such as FGATIR or WAIR (Gravbrot et al., 2020), that may help improve atlas-based registration approaches. We have not investigated the performance of these approaches in this work and this is a question that requires further study. However, their emergence further supports the necessity to augment and improve the longstanding approaches of using atlas-based coordinates, traditionally used for stereotactic targeting. This includes novel MRI sequences to guide atlas-template warping or template-free approaches such as ours. A common thread, linking all of these, is that they should be designed to support, but not replace, clinical decision making, and must be subject to a high standard of scrutiny to fully understand their utility and limitations in the clinical arena.

\section{Conclusions}

The Vim is a key target for the surgical treatment of tremor, yet current approaches using atlas-based coordinates fail to capture interindividual variability. We have shown that tractography-defined Vim localisation is robust to obvious methodological confounds and can effectively capture anatomical variability in vivo. This work provides a foundation for developing non-invasive, translational tools for patient-specific stereotactic targeting in neurosurgery, with the potential to help improve surgical safety, patient comfort and long-term clinical outcomes in these disabling conditions. 


\section{Declaration of Competing Interest}

The authors declare no conflicts of interest, financial or otherwise.

\section{Funding sources}

This work was supported by the Engineering and Physical Sciences Research Council (EPSRC, grants EP/L016476/1 and EP/S021930/1), Brain Research UK, and the University College London Hospitals NIHR Biomedical Research Centre. The Unit of Functional Neurosurgery, National Hospital for Neurology and Neurosurgery, London receives support from the Parkinson's Appeal and the Sainsbury Monument Trust. CL is supported by an MRC Clinician Scientist award (MR/R006504/1). The Wellcome Centre for Human Neuroimaging is supported by core funding from the Wellcome Trust (203147/Z/16/Z)

\section{Supplementary materials}

Supplementary material associated with this article can be found, in the online version, at doi:10.1016/j.neuroimage.2021.118231.

\section{References}

Abhinav, K., Yeh, F.-C., Pathak, S., Suski, V., Lacomis, D., Friedlander, R.M., FernandezMiranda, J.C., 2014. Advanced diffusion MRI fiber tracking in neurosurgical and neurodegenerative disorders and neuroanatomical studies: a review. Biochim. Biophys. Acta Mol. Basis Dis. 1842, 2286-2297. doi:10.1016/j.bbadis.2014.08.002.

Akram, H., Dayal, V., Mahlknecht, P., Georgiev, D., Hyam, J., Foltynie, T., Limousin, P., De Vita, E., Jahanshahi, M., Ashburner, J., Behrens, T., Hariz, M., Zrinzo, L., 2018. Connectivity derived thalamic segmentation in deep brain stimulation for tremor. NeuroImage Clin. doi:10.1016/j.nicl.2018.01.008.

Al-Fatly, B., Ewert, S., Kübler, D., Kroneberg, D., Horn, A., Kühn, A.A., 2019. Connectivity profile of thalamic deep brain stimulation to effectively treat essential tremor. bioRxiv 575209. doi:10.1101/575209.

Alho, 2011. Review of Printed and Electronic Stereotactic Atlases of the Human Brain. IntechOpe.

Ambrosen, K.S., Eskildsen, S.F., Hinne, M., Krug, K., Lundell, H., Schmidt, M.N., van Gerven, M.A.J., Mørup, M., Dyrby, T.B., 2020. Validation of structural brain connectivity networks: the impact of scanning parameters. Neuroimage 204, 116207. doi:10.1016/J.NEUROIMAGE.2019.116207.

Amico, E., Goñi, J., 2018. The quest for identifiability in human functional connectomes. Sci. Rep. 8, 8254. doi:10.1038/s41598-018-25089-1.

Amunts, K., et al., 2000. Interhemispheric asymmetry of the human motor cortex related to handedness and gender. Neuropsychologia doi:10.1016/S0028-3932(99)00075-5, In press.

Amunts, K., et al., 2005. Cytoarchitectonic mapping of the human amygdala, hippocampal region and entorhinal cortex: intersubject variability and probability maps. Anat Embryol (Berl) doi:10.1007/s00429-005-0025-5, In press.

Anderson, J.S., Dhatt, H.S., Ferguson, M.A., Lopez-Larson, M., Schrock, L.E., House, P.A., Yurgelun-Todd, D., 2011. Functional connectivity targeting for deep brain stimulation in essential tremor. AJNR Am. J. Neuroradiol. 32, 1963-1968. doi:10.3174/ajnr.A2638.

Asanuma, C., Thach, W.T., Jones, E.G., 1983. Anatomical evidence for segregated focal groupings of efferent cells and their terminal ramifications in the cerebellothalamic pathway of the monkey. Brain Res. Rev. 5, 267-297. doi:10.1016/0165-0173(83)90016-4.

Ashburner, J., Friston, K.J., 2011. Diffeomorphic registration using geodesic shooting and Gauss-Newton optimisation. Neuroimage 55, 954-959. doi:10.1016/j.neuroimage.2010.12.

Ashburner, J., Friston, K.J., 2005. Unified segmentation. Neuroimage 26, 839-851. doi:10.1016/j.neuroimage.2005.02.018

Baum, G.L., Roalf, D.R., Cook, P.A., Ciric, R., Rosen, A.F.G., Xia, C., Elliott, M.A., Ruparel, K., Verma, R., Tunç, B., Gur, R.C., Gur, R.E., Bassett, D.S., Satterthwaite, T.D., 2018. The impact of in-scanner head motion on structural connectivity derived from diffusion MRI. Neuroimage 173, 275-286. doi:10.1016/j.neuroimage.2018.02.041.

Behrens, T.E.J., Johansen-Berg, H., Woolrich, M.W., Smith, S.M., WheelerKingshott, C.A.M., Boulby, P.A., Barker, G.J., Sillery, E.L., Sheehan, K., Ciccarelli, O., Thompson, A.J., Brady, J.M., Matthews, P.M., 2003. Non-invasive mapping of connections between human thalamus and cortex using diffusion imaging. Nat. Neurosci. 6, 750-757. doi:10.1038/nn1075.

Benabid, A.L., Pollak, P., Hoffmann, D., Gervason, C., Hommel, M., Perret, J.E., de Rougemont, J., Gao, D.M., 1991. Long-term suppression of tremor by chronic stimulation of the ventral intermediate thalamic nucleus. Lancet 337, 403-406. doi:10.1016/0140-6736(91)91175-T.

Benabid, A.L., Pollak, P., Seigneuret, E., Hoffmann, D., Gay, E., Perret, J., 1993. Chronic VIM Thalamic Stimulation in Parkinson's Disease, Essential Tremor and ExtraPyramidal Dyskinesias BT - Advances in Stereotactic and Functional Neurosurgery 10, in: Meyerson, B.A., Broggi, G., Martin-Rodriguez, J., Ostertag, C., Sindou, M. (Eds.). Springer Vienna, Vienna, pp. 39-44.
Benabid, A.L., et al., 1989. Treatment of Parkinson tremor by chronic stimulation of the ventral intermediate nucleus of the thalamus. Rev. Neurol. 145, 320-323.

Bostan, A.C., Dum, R.P., Strick, P.L., 2013. In: Cerebellar Networks With the Cerebral Cortex Nd Basal Ganglia, 17. Trends Cogn. Sci, pp. 241-254. doi:10.1016/j.tics.2013.03.003.

Buchanan, C.R., Bastin, M.E., Ritchie, S.J., Liewald, D.C., Madole, J.W., Tucker-Drob, E.M., Deary, I.J., Cox, S.R., 2020. The effect of network thresholding and weighting on structural brain networks in the UK Biobank. Neuroimage 211, 116443. doi:10.1016/j.neuroimage.2019.116443.

Chen, D.Q., Dell'Acqua, F., Rokem, A., Garyfallidis, E., Hayes, D.J., Zhong, J., Hodaie, M., 2019. Diffusion weighted image co-registration: investigation of best practices. bioRxiv, 864108 doi:10.1101/864108.

Chen, T., Mirzadeh, Z., Chapple, K., Lambert, M., Dhall, R., Ponce, F.A., 2016. Asleep” deep brain stimulation for essential tremor. J. Neurosurg. JNS 124, 1842-1849. doi:10.3171/2015.6.JNS15526.

Coenen, V.A., Allert, N., Mädler, B., 2011. A role of diffusion tensor imaging fiber tracking in deep brain stimulation surgery: DBS of the dentato-rubro-thalamic tract (drt) for the treatment of therapy-refractory tremor. Acta Neurochir. (Wien). 153, 1579-1585. doi:10.1007/s00701-011-1036-z.

Cury, R.G., Fraix, V., Castrioto, A., Pérez Fernández, M.A., Krack, P., Chabardes, S. Seigneuret, E., Alho, E.J.L., Benabid, A.L., Moro, E., 2017. Thalamic deep brain stimulation for tremor in Parkinson disease, essential tremor, and dystonia. Neurology 89. doi:10.1212/WNL.0000000000004295, 1416 LP-1423.

DeLong, M., Wichmann, T., 2009. Update on models of basal ganglia function and dysfunction. Parkinsonism Relat. Disord. 15, S237-S240. doi:10.1016/S1353-8020(09)70822-3.

Duval, C., Daneault, J.-F., Hutchison, W.D., Sadikot, A.F., 2016. A brain network model explaining tremor in Parkinson's disease. Neurobiol. Dis. 85, 49-59. doi:10.1016/j.nbd.2015.10.009.

Gallay, M.N., Jeanmonod, D., Liu, J., Morel, A., 2008. Human pallidothalamic and cerebellothalamic tracts: anatomical basis for functional stereotactic neurosurgery. Brain Struct. Funct. 212, 443-463. doi:10.1007/s00429-007-0170-0.

Gardner, R.C., 2001. Psychological Statistics Using SPSS For Windows. Prentice Hall, Upper Saddle River, N.J.

Glasser, M.F., Sotiropoulos, S.N., Wilson, J.A., Coalson, T.S., Fischl, B., Andersson, J.L., Xu, J., Jbabdi, S., Webster, M., Polimeni, J.R., Van Essen, D.C., Jenkinson, M., WUMinn HCP Consortium, 2013. The minimal preprocessing pipelines for the human connectome project. Neuroimage 80, 105-124. 10.1016/j.neuroimage.2013.04.127

Gravbrot, N., Saranathan, M., Pouratian, N., Kasoff, W.S., 2020. Advanced imaging and direct targeting of the motor thalamus and Dentato-Rubro-thalamic tract for tremor: a systematic review. Stereotact. Funct. Neurosurg. 98, 220-240. doi:10.1159/000507030.

Hariz, M.I., Krack, P., Alesch, F., Augustinsson, L.-E., Bosch, A., Ekberg, R., Johansson, F., Johnels, B., Meyerson, B.A., N'Guyen, J.-P., Pinter, M., Pollak, P., von Raison, F., Rehncrona, S., Speelman, J.D., Sydow, O., Benabid, A.-L., 2008. Multicentre European study of thalamic stimulation for parkinsonian tremor: a 6 year follow-up. J. Neurol. Neurosurg. Psychiatry 79, 694. doi:10.1136/jnnp.2007.118653, LP -699.

Hassler, R., Schaltenbrand, G., Bailey, P., 1959. Introduction to Stereotaxic Operation With an Atlas of the Human Brain, Anatomy of the Thalamus. Georg Thieme Verlag, New York.

Hernandez-Fernandez, M., Reguly, I., Jbabdi, S., Giles, M., Smith, S., Sotiropoulos, S.N., 2019. Using GPUs to accelerate computational diffusion MRI: From microstructure estimation to tractography and connectomes. Neuroimage 188, 598-615. doi:10.1016/j.neuroimage.2018.12.015.

Hernández, M., Guerrero, G.D., Cecilia, J.M., García, J.M., Inuggi, A., Jbabdi, S., Behrens, T.E.J., Sotiropoulos, S.N., 2013. Accelerating fibre orientation estimation from diffusion weighted magnetic resonance imaging using GPUs. PLoS One 8, e61892.

Hirai, T., Jones, E.G., 1989. A new parcellation of the human thalamus on the basis of histochemical staining. Brain Res. Brain Res. Rev 14, 1-34. doi:10.1016/0165-0173(89)90007-6.

Hirai, T., Miyazaki, M., Nakajima, H., Shibazaki, T., Ohye, C., 1983. The correlation between tremor characteristics and the predicted volume of effective lesions in stereotactic nucleus ventralis intermedius thalamotomy. Brain 106, 1001-1018. doi:10.1093/brain/106.4.1001.

Horn, A., Li, N., Dembek, T.A., Kappel, A., Boulay, C., Ewert, S., Tietze, A., Husch, A., Perera, T., Neumann, W.-J., Reisert, M., Si, H., Oostenveld, R., Rorden, C., Yeh, F.-C., Fang, Q., Herrington, T.M., Vorwerk, J., Kühn, A.A., 2019. Lead-DBS v2: towards a comprehensive pipeline for deep brain stimulation imaging. Neuroimage 184, 293316. doi:10.1016/j.neuroimage.2018.08.068.

Huguenard, J.R., McCormick, D.A., 2007. Thalamic synchrony and dynamic regulation of global forebrain oscillations. Trends Neurosci. 30, 350-356. doi:10.1016/j.tins.2007.05.007.

Iglesias, J.E., Insausti, R., Lerma-Usabiaga, G., Bocchetta, M., Van Leemput, K., Greve, D.N., van der Kouwe, A., Fischl, B., Caballero-Gaudes, C., Paz-Alonso, P.M., 2018. A probabilistic atlas of the human thalamic nuclei combining ex vivo MRI and histology. Neuroimage 183, 314-326. doi:10.1016/J.NEUROIMAGE.2018.08.012.

Ilinsky, I., Horn, A., Paul-Gilloteaux, P., Gressens, P., Verney, C., KultasIlinsky, K., 2018. Human motor thalamus reconstructed in 3D from continuous sagittal sections with identified subcortical afferent territories. eNeuro 5 . doi:10.1523/ENEURO.0060-18.2018, ENEURO.0060-18.2018.

Jones, E.G., 2007. The Thalamus. Cambridge University Press, Cambridge.

Jones, E.G., 1985. The Thalamus. Plenum, New York.

King, M., Hernandez-Castillo, C.R., Poldrack, R.A., Ivry, R.B., Diedrichsen, J., 2019. Functional boundaries in the human cerebellum revealed by a multi-domain task battery. Nat. Neurosci. 22, 1371-1378. doi:10.1038/s41593-019-0436-x. 
King, N.K.K., Krishna, V., Basha, D., Elias, G., Sammartino, F., Hodaie, M., Lozano, A.M., Hutchison, W.D., 2017. Microelectrode recording findings within the tractography-defined ventral intermediate nucleus. J. Neurosurg. JNS 126, 16691675. doi:10.3171/2016.3.JNS151992.

Kuo, J.-S., Carpenter, M.B., 1973. Organization of pallidothalamic projections in the rhesus monkey. J. Comp. Neurol. 151, 201-235. doi:10.1002/cne.901510302.

Lambert, C., Simon, H., Colman, J., Barrick, T.R., 2017. Defining thalamic nuclei and topographic connectivity gradients in vivo. In: Neuroimage, 158, pp. 466-479. doi:10.1016/j.neuroimage.2016.08.028.

Le Bihan, D., Poupon, C., Amadon, A., Lethimonnier, F., 2006. Artifacts and pitfalls in diffusion MRI. J. Magn. Reson. Imaging 24, 478-488. doi:10.1002/jmri.20683.

Li, L., Rilling, J.K., Preuss, T.M., Glasser, M.F., Hu, X., 2012. The effects of connection reconstruction method on the interregional connectivity of brain networks via diffusion tractography. Hum. Brain Mapp. 33, 1894-1913. doi:10.1002/hbm.21332.

Ling, J., Merideth, F., Caprihan, A., Pena, A., Teshiba, T., Mayer, A.R., 2012. Head injury or head motion? Assessment and quantification of motion artifacts in diffusion tensor imaging studies. Hum. Brain Mapp. 33, 50-62. doi:10.1002/hbm.21192.

Llinás, R., Urbano, F.J., Leznik, E., Ramírez, R.R., van Marle, H.J.F., 2005. Rhythmic and dysrhythmic thalamocortical dynamics: GABA systems and the edge effect. Trends Neurosci 28, 325-333. doi:10.1016/j.tins.2005.04.006.

Llinas, R.R., 1988. The intrinsic electrophysiological properties of mammalian neurons: insights into central nervous system function. Science (80) 242. doi:10.1126/science.3059497, 1654 LP -1664.

Mai, J.K., Forutan, F., 2012. Chapter 19 - Thalamus, in: Mai, J.K., Paxinos, G.B.T.T.H.N.S. (Third E. (Eds.), Academic Press, San Diego, pp. 618-677. 10.1016/B978-012-374236-0.10019-7

Maier-Hein, K.H., Neher, P.F., Houde, J.C., Côté, M.A., Garyfallidis, E., Zhong, J., Chamberland, M., Yeh, F.C., Lin, Y.C., Ji, Q., Reddick, W.E., Reina, J.E., Galvis, J., Thompson, P.M., Requejo, F.D.S., Laguna, P.L., Lacerda, L.M., Barrett, R., Dell'Acqua, F., Catani, M., Petit, L., Caruyer, E., Daducci, A., Dyrby, T.B., Holland-Letz, T., Hilgetag, C.C., Stieltjes, B., Descoteaux, M.Glass, J.O., Chen, D.Q., Feng, Y., Gao, C., Wu, Y., Ma, J., He, R., Li, Q., Westin, C.-F., Deslauriers-Gauthier, S., González, J.O.O., Paquette, M., St-Jean, S., Girard, G., Rheault, F., Sidhu, J., Tax, C.M.W., Guo, F., Mesri, H.Y., Dávid, S., Froeling, M., Heemskerk, A.M., Leemans, A., Boré, A., Pinsard, B., Bedetti, C., Desrosiers, M., Brambati, S., Doyon, J., Sarica, A., Vasta, R., Cerasa, A., Quattrone, A., Yeatman, J., Khan, A.R., Hodges, W., Alexander, S., Romascano, D., Barakovic, M., Auría, A., Esteban, O., Lemkaddem, A., Thiran, J.-P., Cetingul, H.E., Odry, B.L., Mailhe, B., Nadar, M.S., Pizzagalli, F., Prasad, G., Villalon, 2017. The challenge of mapping the human connectome based on diffusion tractography. Nat. Commun. 8, 1349. doi:10.1038/s41467-017-01285-x.

Mancini, M., Vos, S.B., Vakharia, V.N., O'Keeffe, A.G., Trimmel, K., Barkhof, F., Dorfer, C., Soman, S., Winston, G.P., Wu, C., Duncan, J.S., Sparks, R., Ourselin, S., 2019. Automated fiber tract reconstruction for surgery planning: extensive validation in language-related white matter tracts. NeuroImage Clin. 23, 101883. doi:10.1016/j.nicl.2019.101883.

Melo, et al., 2021. Comparison of direct MRI guided versus atlas-based targeting for subthalamic nucleus and globus pallidus deep brain stimulation. $\mathrm{Br} \mathrm{J}$ Neurosurg. doi:10.1080/02688697.2020.1850641, In press

Middlebrooks, E.H., Holanda, V.M., Tuna, I.S., Deshpande, H.D., Bredel, M., Almeida, L., Walker, H.C., Guthrie, B.L., Foote, K.D., Okun, M.S., 2018a. A method for preoperative single-subject thalamic segmentation based on probabilistic tractography for essential tremor deep brain stimulation. Neuroradiology 60, 303-309. doi:10.1007/s00234-017-1972-2

Morel, A., Magnin, M., Jeanmonod, D., 1997. Multiarchitectonic and stereotactic atlas of the human thalamus. J. Comp. Neurol. 387, 588-630. doi:10.1002/(SICI)1096-9861(19971103)387:4<588::AID-CNE8 > 3.0.CO;2-Z.

Muller, J., Alizadeh, M., Li, L., Thalheimer, S., Matias, C., Tantawi, M., Miao, J., Silverman, M., Zhang, V., Yun, G., Romo, V., Mohamed, F.B., Wu, C., 2020. Feasibility of diffusion and probabilistic white matter analysis in patients implanted with a deep brain stimulator. NeuroImage Clin. 25, 102135. doi:10.1016/j.nicl.2019.102135.

Muthuraman, M., Heute, U., Arning, K., Anwar, A.R., Elble, R., Deuschl, G., Raethjen, J., 2012. Oscillating central motor networks in pathological tremors and voluntary movements. What makes the difference? Neuroimage 60, 1331-1339. doi:10.1016/j.neuroimage.2012.01.088

Nimsky, C., Bauer, M., Carl, B., 2016. Merits and limits of tractography techniques for the uninitiated. Adv. Tech. Stand. Neurosurg. 37-60. doi:10.1007/978-3-21359-0 2.

Nowacki, A., Schlaier, J., Debove, I., Pollo, C., 2018. Validation of diffusion tensor imaging tractography to visualize the dentatorubrothalamic tract for surgical planning. J. Neurosurg. JNS 130, 99-108. doi:10.3171/2017.9.JNS171321.

Nowinski, W.L., Belov, D., Thirunavuukarasuu, A., Benabid, A.L., 2005. A probabilistic functional atlas of the VIM nucleus constructed from pre-, intra- and postoperative electrophysiological and neuroimaging data acquired during the surgical treatment of Parkinson's disease patients. Stereotact. Funct. Neurosurg. 83, 190-196. doi:10.1159/000091082.

Palesi, F., De Rinaldis, A., Castellazzi, G., Calamante, F., Muhlert, N., Chard, D., Tournier, J.D., Magenes, G., D’Angelo, E., Gandini Wheeler-Kingshott, C.A.M., 2017. Contralateral cortico-ponto-cerebellar pathways reconstruction in humans in vivo: implications for reciprocal cerebro-cerebellar structural connectivity in motor and nonmotor areas. Sci. Rep. 7, 12841. doi:10.1038/s41598-017-13079-8.

Palesi, F., Tournier, J.-D., Calamante, F., Muhlert, N., Castellazzi, G., Chard, D., D'Angelo, E., Wheeler-Kingshott, C.A.M., 2015. Contralateral cerebello-thalamocortical pathways with prominent involvement of associative areas in humans in vivo. Brain Struct. Funct. 220, 3369-3384. doi:10.1007/s00429-014-0861-2.

Papavassiliou, E., Rau, G., Heath, S., Abosch, A., Barbaro, N.M., Larson, P.S., Lamborn, K., Starr, P.A., 2004. Thalamic deep brain stimulation for essential tremor: relation of lead location to outcome. In: Neurosurgery, 54, pp. 1120-1130. doi:10.1227/01.NEU.0000119329.66931.9E.

Petersen, M.V, Lund, T.E., Sunde, N., Frandsen, J., Rosendal, F., Juul, N., Østergaard, K., 2017. Probabilistic versus deterministic tractography for delineation of the corticosubthalamic hyperdirect pathway in patients with Parkinson disease selected for deep brain stimulation. J. Neurosurg. JNS 126.

Pollak, P., Benabid, A.L., Gervason, C.L., Hoffmann, D., Seigneuret, E., 1993. Long-term effects of chronic stimulation of the ventral intermediate thalamic nucleus in different types of tremor. Adv. Neurol. 60, 408-413.

Pouratian, N., Zheng, Z., Bari, A.A., Behnke, E., Elias, W.J., DeSalles, A.A.F., 2011. Multi-institutional evaluation of deep brain stimulation targeting using probabilistic connectivity-based thalamic segmentation. J. Neurosurg. JNS 115, 995-1004. doi:10.3171/2011.7.JNS11250.

Raethjen, J., Deuschl, G., 2012. The oscillating central network of essential tremor. Clin. Neurophysiol. 123, 61-64. doi:10.1016/j.clinph.2011.09.024.

Sakai, S.T., 2013. Cerebellar Thalamic and Thalamocortical Projections BT - Handbook of the Cerebellum and Cerebellar Disorders, in: Manto, M., Schmahmann, J.D., Rossi, F., Gruol, D.L., Koibuchi, N. (Eds.), Springer Netherlands, Dordrecht, pp. 529-547. 10.1007/978-94-007-1333-8_24

Sammartino, F., Krishna, V., King, N.K.K., Lozano, A.M., Schwartz, M.L., Huang, Y., Hodaie, M., 2016. Tractography-based ventral intermediate nucleus targeting: novel methodology and intraoperative validation. Mov. Disord. 31, 1217-1225. doi:10.1002/mds.26633.

Schaltenbrand, G., 1977. Atlas for Stereotaxy of the Human Brain. Georg Thieme.

Steriade, M., Llinás, R.R., 1988. The functional states of the thalamus and the associated neuronal interplay. In: Physiol. Rev., 68, pp. 649-742. doi:10.1152/physrev.1988.68.3.649.

Strick, P.L., 1976. Activity of ventrolateral thalamic neurons during arm movement. J. Neurophysiol. 39, 1032-1044. doi:10.1152/jn.1976.39.5.1032.

Strick, P.L., Dum, R.P., Fiez, J.A., 2009. Cerebellum and nonmotor function. Annu. Rev. Neurosci. 32, 413-434. doi:10.1146/annurev.neuro.31.060407.125606.

Talairach, J., 1988. Co-Planar Stereotaxic Atlas of the Human Brain-3-Dimensional Proportional System: An Approach to Cerebral Imaging. Thieme.

Tian, Q., Wintermark, M., Jeffrey Elias, W., Ghanouni, P., Halpern, C.H., Henderson, J.M., Huss, D.S., Goubran, M., Thaler, C., Airan, R., Zeineh, M., Pauly, K.B., McNab, J.A., 2018. Diffusion MRI tractography for improved transcranial MRI-guided focused ultrasound thalamotomy targeting for essential tremor. NeuroImage Clin. 19, 572-580. doi:10.1016/j.nicl.2018.05.010.

Tijssen, R.H.N., Jansen, J.F.A., Backes, W.H., 2009. Assessing and minimizing the effects of noise and motion in clinical DTI at 3 T. Hum. Brain Mapp. 30, 2641-2655. doi:10.1002/hbm.20695.

Traynor, C., Heckemann, R.A., Hammers, A., O'Muircheartaigh, J., Crum, W.R., Barker, G.J., Richardson, M.P., 2010. Reproducibility of thalamic segmentation based on probabilistic tractography. Neuroimage 52, 69-85. doi:10.1016/j.neuroimage.2010.04.024.

Tsai, S.-Y., 2018. Reproducibility of structural brain connectivity and network metrics using probabilistic diffusion tractography. Sci. Rep. 8, 11562. doi:10.1038/s41598-018-29943-0

Tsolaki, E., Downes, A., Speier, W., Elias, W.J., Pouratian, N., 2017. The potential value of probabilistic tractography-based for MR-guided focused ultrasound thalamotomy for essential tremor. NeuroImage Clin. 17, 1019-1027. doi:10.1016/j.nicl.2017.12.018.

Tsuboi, Y., Slowinski, J., Josephs, K.A., Honer, W.G., Wszolek, Z.K., Dickson, D.W., 2003. Atrophy of superior cerebellar peduncle in progressive supranuclear palsy. Neurology 60. doi:10.1212/01.WNL.0000068011.21396.F4, 1766 LP -1769.

Van Essen, D.C., Smith, S.M., Barch, D.M., Behrens, T.E.J., Yacoub, E., Ugurbil, K., Consortium, W.-M.H.C.P., 2013. The WU-Minn Human connectome project: an overview. Neuroimage 80, 62-79. doi:10.1016/j.neuroimage.2013.05.041.

Van Essen, D.C., Ugurbil, K., Auerbach, E., Barch, D., Behrens, T.E.J., Bucholz, R., Chang, A., Chen, L., Corbetta, M., Curtiss, S.W., Della Penna, S., Feinberg, D., Glasser, M.F., Harel, N., Heath, A.C., Larson-Prior, L., Marcus, D., Michalareas, G., Moeller, S., Oostenveld, R., Petersen, S.E., Prior, F., Schlaggar, B.L., Smith, S.M., Snyder, A.Z., Xu, J., Yacoub, E.WU-Minn HCP Consortium, 2012. The human connectome project: a data acquisition perspective. Neuroimage 62, 2222-2231. doi:10.1016/j.neuroimage.2012.02.018.

Vitek, J.L., Ashe, J., DeLong, M.R., Alexander, G.E., 1994. Physiologic properties and somatotopic organization of the primate motor thalamus. J. Neurophysiol. 71, 14981513. doi:10.1152/jn.1994.71.4.1498.

Vitek, J.L., Ashe, J., DeLong, M.R., Kaneoke, Y., 1996. Microstimulation of primate motor thalamus: somatotopic organization and differential distribution of evoked motor responses among subnuclei. J. Neurophysiol. 75, 2486-2495. doi:10.1152/jn.1996.75.6.2486.

Wang, D., Buckner, R.L., Liu, H., 2013. Cerebellar asymmetry and its relation to cerebral asymmetry estimated by intrinsic functional connectivity. J. Neurophysiol. 109, 4657. doi:10.1152/jn.00598.2012.

Zalesky, A., Fornito, A., Cocchi, L., Gollo, L.L., van den Heuvel, M.P., Breakspear, M., 2016. Connectome sensitivity or specificity: which is more important? Neuroimage 142, 407-420. doi:10.1016/j.neuroimage.2016.06.035.

Zhang, H., Yushkevich, P.A., Alexander, D.C., Gee, J.C., 2006. Deformable registration of diffusion tensor MR images with explicit orientation optimization. Med. Image Anal. 10, 764-785. doi:10.1016/j.media.2006.06.004.

Zrinzo, L., Foltynie, T., Limousin, P., Hariz, M.I., 2012. Reducing hemorrhagic complications in functional neurosurgery: a large case series and systematic literature review. J. Neurosurg. 116, 84-94. doi:10.3171/2011.8.JNS101407, JNS 